\title{
Machine Learning Approaches for Predicting Geometric and Mechanical Characteristics for Single P420 Laser Beads Clad onto an AISI 1018 Substrate
}

Bita Mohajernia ( $\nabla$ bita.mohajernia@gmail.com )

University of Windsor Faculty of Engineering https://orcid.org/0000-0001-7119-2490

Seyedeh Elnaz Mirazimzadeh

University of Windsor Faculty of Engineering

Alireza Pasha

University of Windsor Faculty of Engineering

Ruth Jill Urbanic

University of Windsor Faculty of Engineering https://orcid.org/0000-0002-2906-7618

\section{Research Article}

Keywords: Additive Manufacturing, Metal, Direct Energy Deposition, Experimental, Simulation, Artificial Neural Network, Adaptive Neuro Fuzzy Inference System, 420 Stainless Steel, Hardness, Residual Stress

Posted Date: July 12th, 2021

DOI: https://doi.org/10.21203/rs.3.rs-682870/v1

License: (c) (1) This work is licensed under a Creative Commons Attribution 4.0 International License. Read Full License

Version of Record: A version of this preprint was published at The International Journal of Advanced Manufacturing Technology on October 16th, 2021. See the published version at https://doi.org/10.1007/s00170-021-08155-3. 


\title{
Machine Learning Approaches for Predicting Geometric and Mechanical Characteristics for Single P420 Laser Beads Clad onto an AISI 1018 Substrate
}

\author{
Bita Mohajernia $^{\text {a }}$, Seyedeh Elnaz Mirazimzadeh ${ }^{\mathrm{a}}$, Alireza Pasha ${ }^{\mathrm{a}}$, and R. Jill Urbanic ${ }^{\text {a+ }}$ \\ ${ }^{a}$ Department of Mechanical, Automotive, and Materials Engineering, University of Windsor, Windsor, \\ Ontario, Canada N9B $3 P 4$
}

phone: 519-253-3000, ext. 2633

fax: (519) 973-7062

Email address jurbanic@uwindsor.ca

+ corresponding author

\begin{abstract}
For effective bead deposition based additive manufacturing (AM) processes such as directed energy deposition, the final mechanical and physical properties should be predicted in tandem with the bead geometry characteristics. Experimental approaches to investigate the final geometry and the mechanical properties are costly, and simulation solutions are timeconsuming. Alternative artificial intelligent (AI) systems are explored as they are a powerful approach to predict such properties. In the present study, the geometrical properties, as well as the mechanical properties (residual stress and hardness) for single bead clads are investigated. Experimental data is used to calibrate multi-physics finite element models, and both data sets are used to seed the AI models. The adaptive neuro-fuzzy inference system (ANFIS) and a feed-forward back-propagation artificial neural network (ANN) system are utilized to explore their effectiveness in the 1D (discrete values), 2D (cross sections), and 3D (complete bead) domains. The prediction results are evaluated using the mean relative error measure. The ANFIS predictions are more precise than those from the ANN for the 1D and 2D domains, but the ANN had less error for the 3D scenario. These models are capable of predicting the geometrical and the mechanical properties values very well, including capturing the mechanical properties in transient regions; however, this research should be extended for multibead scenarios before a conclusive 'best approach' strategy can be determined.
\end{abstract}


Keywords: Additive Manufacturing; Metal; Direct Energy Deposition; Experimental; Simulation; Artificial Neural Network; Adaptive Neuro Fuzzy Inference System; 420 Stainless Steel, Hardness; Residual Stress

\section{Introduction}

\subsection{Additive Manufacturing}

Additive manufacturing is the process of building parts from a computer-aided design (CAD) model by successively adding material layer by layer, realizing the part with minimal excess material. Usually, a heat source is applied to melt or cure the raw materials as they are being formed into the final component shape. Conversely, conventional fabrication methods for objects by removing material via milling or other machining processes introduces much waste, but there is no significant heat introduced into the process. There are seven main categories of AM technologies including vat photopolymerization, material jetting, binder jetting, material extrusion, powder bed fusion, directed energy deposition, and sheet lamination [1]. The directed energy deposition method, which is the focus of this research, is one of the metallic additive manufacturing processes where a machine tool or a robot with a deposition nozzle traverses around an object and deposits metal powder onto existing surfaces. Material is melted using a laser, electron beam or plasma arc upon deposition [1].

\subsection{Directed Energy Deposition Additive Manufacturing}

Directed Energy Deposition (DED) is a subset of the additive manufacturing process family. It is a metal additive process in which blown powder or a wire is fed through a nozzle, and a power source or energy type is introduced to melt the material, and the beads are deposited onto a layer or substrate. Components can be repaired as well as built up using DED processes. 
Laser clad overlay operations are in the DED domain, and usually utilized for coating surfaces to improve the performance of the surface, or to repair components such as molds. In this process, a laser beam melts the material while it is being distributed onto a surface. A thin layer on the surface of the substrate melts to form a bond between the clad and the substrate, this is the dilution zone (Figure 1). This research focuses on single bead depositions of 420 stainless steel onto a mild steel substrate. The input parameters for a laser cladding operation play a significant role in the quality of the bead. As a result, selecting and controlling the input parameters to achieve the desired results is a concern for the manufacturers.

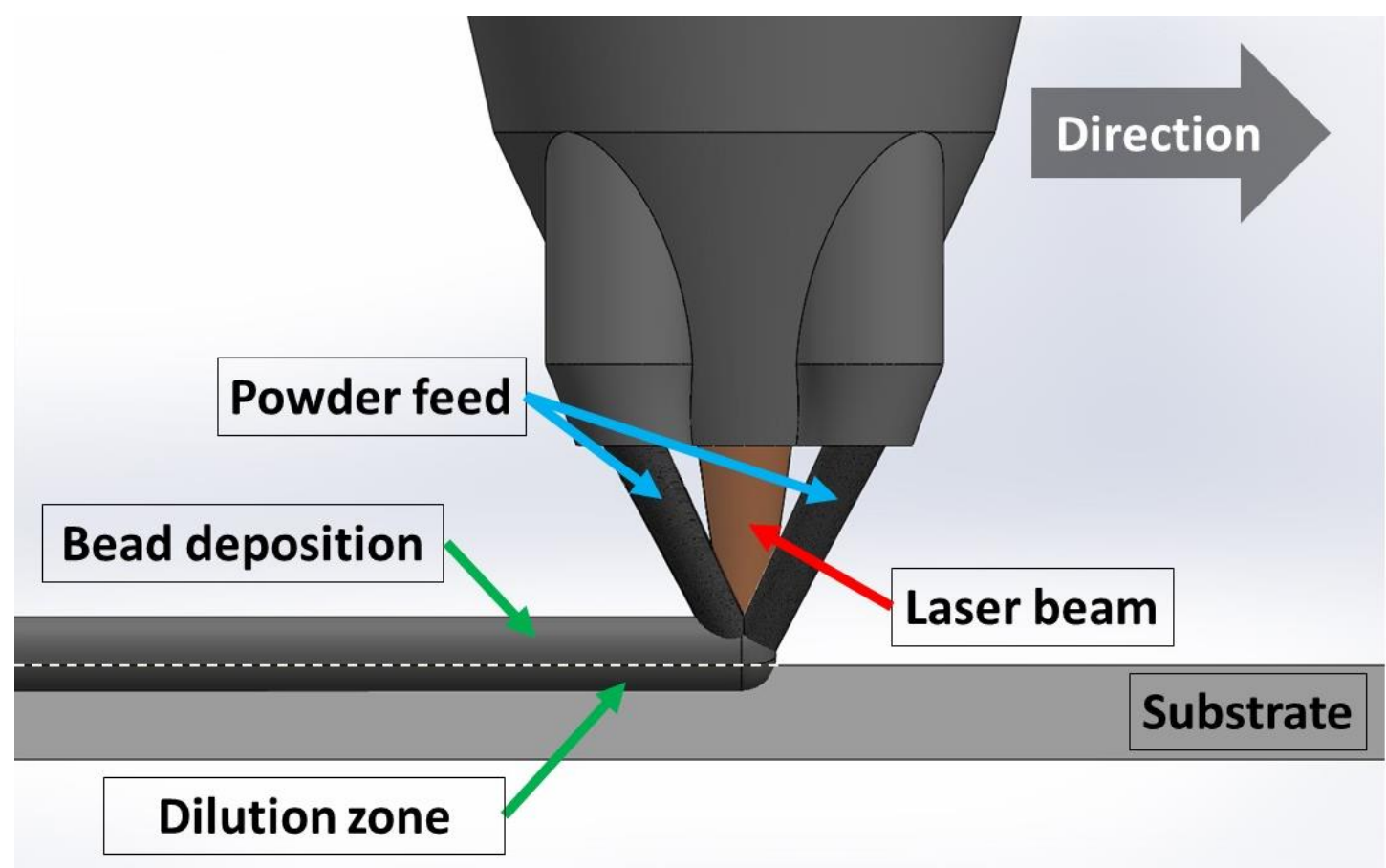

Figure 1. Schematic diagram of laser cladding process

Each process parameter in laser cladding process including the power, travel speed, material feed rate, the contact tip to work piece distance, and the focal length has a distinct effect on the geometry and the mechanical properties of the bead [2][3][4][5][6]. Several experimental investigations have been found in the literature review to analyse the effect of process parameters on clad bead geometry and clad mechanical characteristics. Chen et al investigated the effects of the process parameters including laser power, scanning speed, pre-placed powder 
thickness, laser spot diameter and multi-track overlapping ratio on the quality characteristics of the ceramic coatings on Ti6Al4V substrates. Using L27(313) orthogonal arrays designed with Taguchi method, they conducted multi-track cladding experiments to investigate the geometric properties and microhardness of coatings [6]. Another study investigated the effects of varying percentages of overlap between multiple beads, ranging from 30 to 47 percent. Using experimental measurements, they showed that the percentage overlap impacts the hardness and the depth of the melt pool [7]. In another study conducted by Zhao et al., a single factor experiment with 125 groups was conducted to investigate the impact of process parameters on the cross-sectional area of the YCF104 clad track. It has been found the height of the clad track is largely determined by scan speed, while laser power is the most significant factor for determining the width and depth of the heat-affected zone [8].

Understanding the process parameter to geometric relationships are important for process planning scenarios, but the mechanical and physical properties also need to be considered, and prediction models are required for effective process planning. Due to the high thermal gradients and the rapid solidification rate, the generation of residual stresses with high magnitudes can occur. The high amount of residual stress leads to the non-uniform plastic deformation of the plate, and the bead geometry. This is one of the most important issues when analysing the mechanical properties of the bead. Residual stresses could lead to cracks within the piece in addition to undesirable distortion; therefore, it is important to achieve a laser clad bead with a minimum amount of residual stress. Therefore, the process parameters play an important role in the magnitude of the induced residual stress, the distortion development, the final mechanical properties, and the shape of the bead.

Finite Element Analysis (FEA) and analytical models are also utilized to predict the mechanical properties as well. Mirkoohi et al. [9], 2020 proposed a thermomechanical analytical model to predict the in-process elastoplastic hardening thermal stress and strain which can model the 
thermal stress of a single track either in powder bed systems such as laser powder bed fusion (LPBF) and powder feed systems such as directed metal deposition (DMD). Nazemi and Urbanic [10] proposed a three-dimensional finite element model (FEM) for a powder-feed laser cladding process to predict the mechanical and physical properties, but the geometry needed to be predefined and the simulation approaches were computationally costly for simple single bead and linear multiple bead case studies. For more complex and realistic components, the simulations might take weeks or months of processing time. Several researchers have explored the residual stress formation and its pattern using FEA method for the LPBF AM process $[11][12][13]$; however, the high computational cost is considerable. Therefore, a hybrid approach where data fusion between experimental, simulation and machine learning strategies are being investigated for predicting the results for DED processes. Machine learning is a tool for using data that includes a variety of conditions, followed by an implicit relationship between inputs and outputs. Thus, with use of machine learning the impacts of the process parameters on the mechanical and geometrical properties of the parts can be directly obtained in computationally efficient manner with no need of solving the mechanical equilibrium equations. Presently, the machine learning techniques are becoming popular in the field of material science and manufacturing.

Machine learning approach has been widely implemented to investigate and predict the geometry of the bead. A range of process parameters implemented in various deposition methods are used as an input to generate and train the mathematical model and predict the geometrical data [14], [15].

The thermal profile of the deposited part was predicted by Ren et al.in 2019. They implemented recurrent neural network and also deep neural network to correlate between the toolpath (laser scan pattern) and thermal profile of the deposited part. They used finite element simulation for 
data generation and introduced a unique dataset structure to train the neural network based on the geometry of the part and the laser scanning strategies [16].

Mechanical properties including tensile and compressive stresses were calculated using an Artificial Neural Network as a tool to link the process parameter such as layer thickness, orientation, raster angle, raster width, and air gap [17][18].

Wu et al, in 2020 predicted residual stresses considering four process parameters including arc power, scanning speed, substrate preheat temperature and substrate thickness in wire-arc additive manufacturing. In their approach, arc power, scanning speed, substrate preheat temperature and substrate thickness are the inputs and longitudinal residual stress of the deposition centre point is the output of the model. Both algorithms predict residual stresses with $97 \%$ accuracy [19].

Residual stress profiles in stainless steel pipe girth welds were predicted by developing the artificial neural network (ANN) and adaptive neuro fuzzy inference system (ANFIS). The performance of models was evaluated. It was concluded that the ANN trained using LevenbergMarquadt, and ANFIS based on a hybrid algorithm, were far superior to ANN model trained by resilient-backpropagation and ANFIS using backpropagation method [20].

Although some research has been performed in the welding domain, there is a lack of research related to a performing comprehensive analysis which considers the effects of the laser cladding input parameters on both the geometrical and mechanical characteristics of a laser clad bead simultaneously. The goal of this research is to evaluate the effectiveness of a machine learning (ML) approach for predicting the bead geometry (a discrete value), and selected mechanical and physical characteristics, which can vary throughout the bead. Residual stresses are emphasized in this research as they can lead to undesirable distortion and cracks. We need to: (i) understand the residual stress characteristics, (ii) link them to the bead geometry and input parameters, and (iii) develop predictive models. Hardness is also considered. The types 
of effective predictive models need to be determined; therefore, two ML strategies are utilized. Data fusion approaches are applied to generate data sets for these analyses. This is described in the next section.

\section{Research Methodology}

The research methodology consists of two main steps: (i) data collection, and (ii) machine learning based predictive model development. The process flow is shown in Figure 2. Geometrical, Vickers microhardness and residual stress characteristics were collected for single laser clad beads of P420 stainless steel powder deposited onto low alloyed carbon steel plates for a wide range of process settings. A coaxial powder injection laser cladding process was employed for the experimental activities. In addition to the data from the experiment sets, calibrated simulation models are developed to seed the ML based mathematical models. A multi-perspective analysis has been performed by using the Artificial Neural Networks (ANN) and the Adaptive Neuro Fuzzy Inference System (ANFIS) models to predict geometric and mechanical properties. Both the ANN and ANFIS models are validated using the experimental and numerical data. The performance of ANFIS and ANN approaches to predict the residual stresses is also compared.

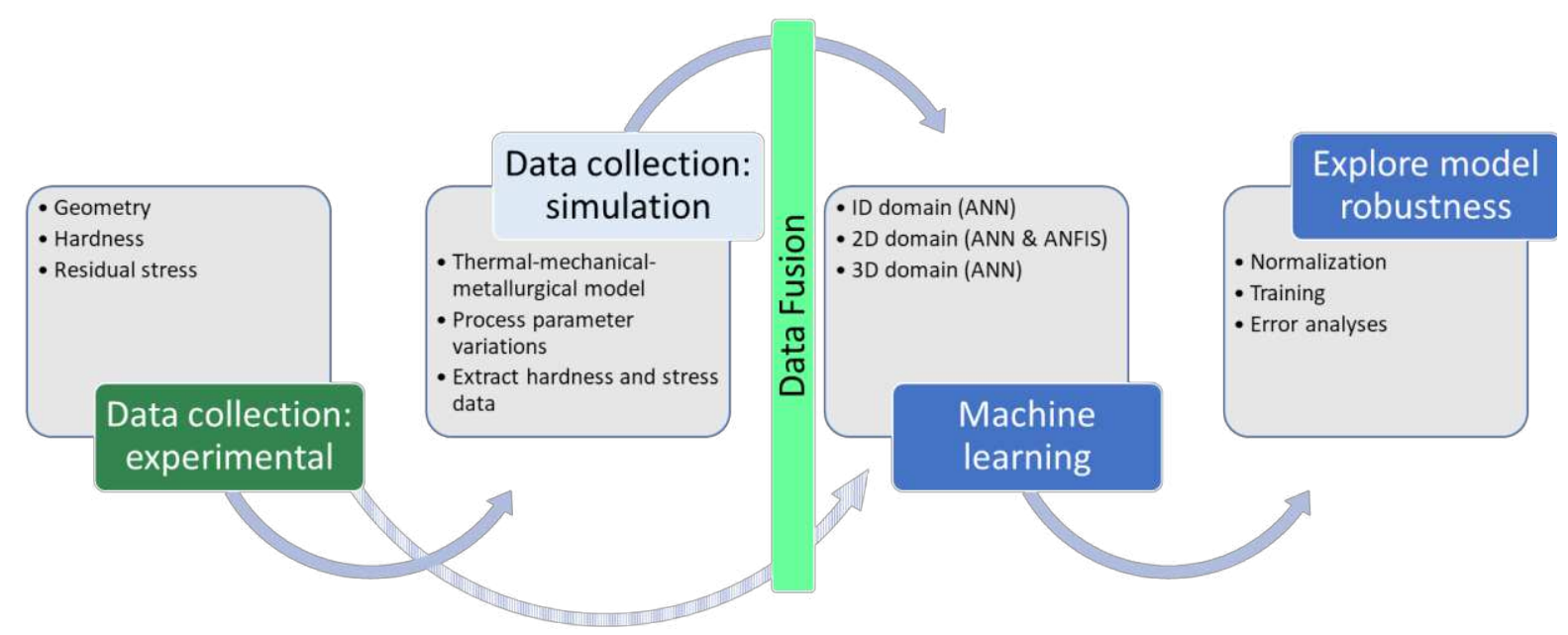

Figure 2. The general process flow for this research 
This study has comprehensively assessed ML prediction strategies for three different domains: i) the $1 \mathrm{D}$ domain in which both the geometrical and properties are to be predicted by an ANN and ANFIS model for discrete geometry and mechanical characteristics, ii) the 2D domain, where the residual stress and hardness along the middle cross section of the laser clad bead and the substrate are predicted by the ANNs and ANFIS models, iii) the 3D domain, where the residual stress and hardness are predicted throughout the bead considering the entire bead geometry and the substrate using the ANNs and ANFIS models. The 1D and 2D models contain simplifying assumptions to provide an initial performance overview for predicting cladding bead characteristics. For the 1D approach, the average values are considered for hardness, and maximum and minimum values are considered for the residual stress. This reduces the computational cost significantly, but it should be noted that the average value does not necessarily represent critical information. A 2D-based approach was considered to establish initial relationships between variable residual stresses, locations, and the process parameters. However, this consideration is limited to the assumption that the thermal gradients occur in the depth direction only. In the 2D model, it is assumed that the thermal gradient is constant along the bead and has no effect on the induced residual stresses. However, in reality the residual stress varies throughout the bead. Consequently, an extended approach considering the variable data along the bead length has been investigated. The 3D model explores a comprehensive big data predictive solution that increases the data collection time and computational cost. However, the residual stress (or hardness) can be predicted in each point within the bead, including the start-stop transient zones. Therefore, critical information can be predicted with confidence. Overall, deep learning methods are applied in all mentioned models which reduces the computing time. Figure 3 illustrates the domains being considered for this research. The yellow dots demonstrate the measuring points in a single laser clad bead for residual stress. 


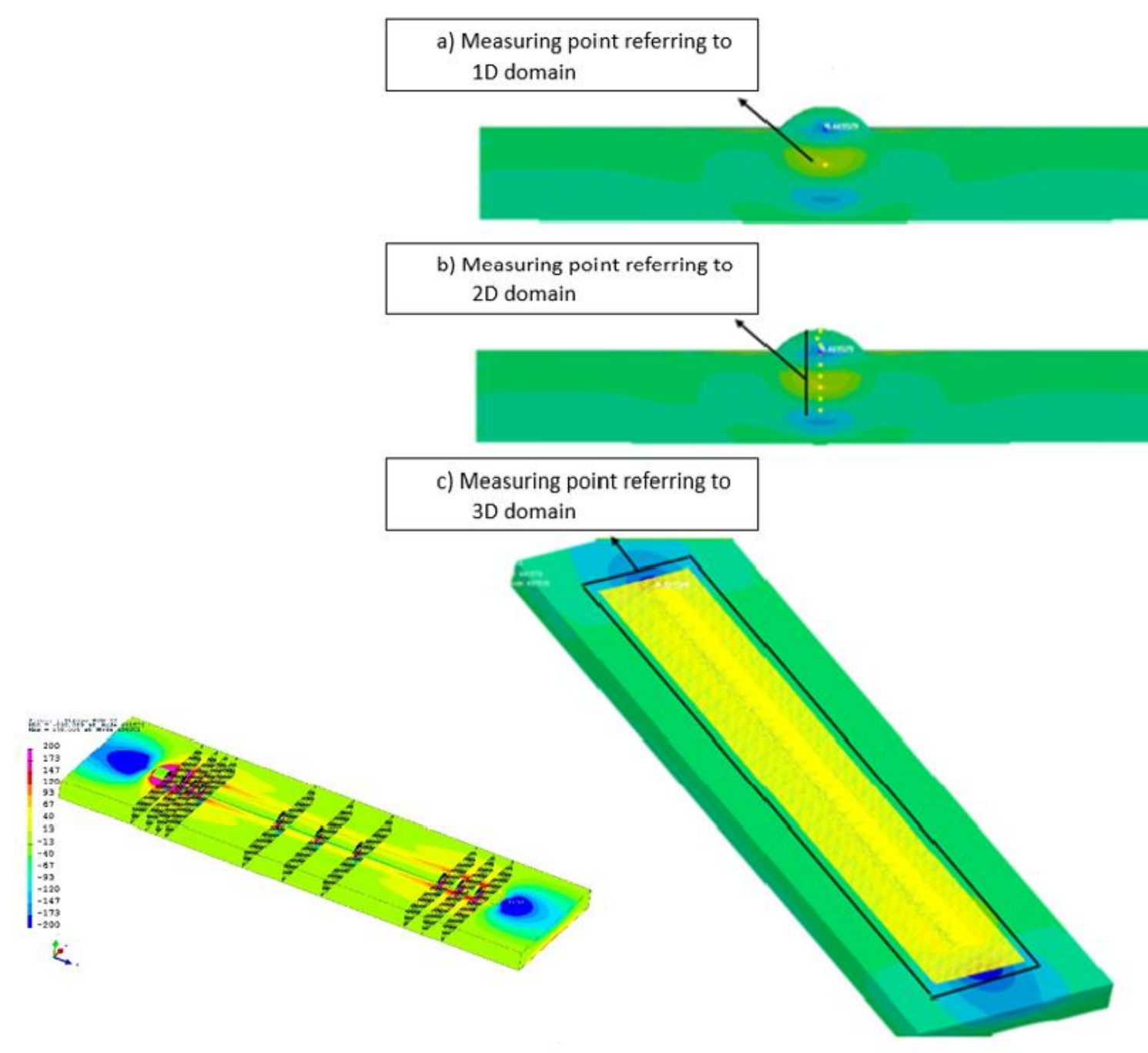

Figure 3. Demonstration of the studied domain of a single laser clad bead - note the variations of the residual stress for different slice planes orthogonal to the bead a)1D domain (maximum compressive stress value), b) 2D domain, c) 3D domain

\subsection{Experimental setup}

Single-pass bead sets of P420 were deposited onto AISI 1018. A comprehensive design of experiments approach was taken to explore five process parameters at five different levels [21]. A coaxial deposition head, which was mounted onto 6 serial axis robots employing a 4kW diode laser, was employed to deposit the clad beads. Three replicates were performed for each 
experiment. Argon gas was employed to protect the melt pool from the atmosphere and was the conveying media for the powder. The process parameters are listed in Table 1.

\begin{tabular}{|c|c|c|c|c|c|c|c|c|c|c|}
\hline & A & B & C & D & $\mathbf{E}$ & $\mathbf{F}$ & G & $\mathbf{H}$ & I & $\mathbf{J}$ \\
\hline Powder feed rate (g/min) & 20 & 15 & 15 & 15 & 30 & 20 & 20 & 20 & 20 & 20 \\
\hline Laser power $(\mathrm{KW})$ & 2.5 & 2 & 3 & 2 & 2.5 & 2.5 & 4 & 2.5 & 2.5 & 2.5 \\
\hline Focal length of lens ( $\mathrm{mm})$ & 400 & 390 & 410 & 390 & 400 & 400 & 400 & 400 & 420 & 400 \\
\hline Laser speed $(\mathrm{mm} / \mathrm{S})$ & 10 & 7.5 & 7.5 & 12.5 & 10 & 10 & 10 & 15 & 10 & 10 \\
\hline Contact tip to work piece distance $(\mathrm{mm})$ & 21 & 24 & 24 & 22 & 23 & 23 & 23 & 23 & 23 & 25 \\
\hline
\end{tabular}

Table 1.Input parameters for the single bead specimens

The metallographic operations i.e., grinding and polishing of the cross-sectional samples were done manually according to the Stuers application notes for the stainless-steel materials [21]. The observations were performed using a Leica Q5501W light microscope. The bead width, depth of penetration, and height of the beads were measured using Image-pro Plus software. Figure 4 shows the details of the geometry measurements.

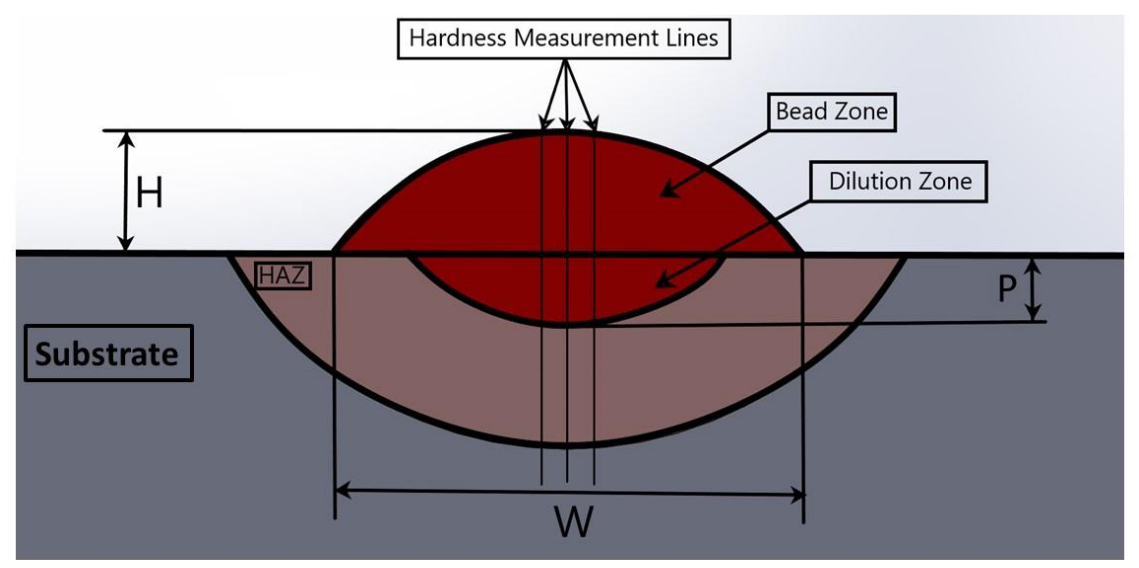

Figure 4.Geometry measurements and hardness measurement line 
The micro-hardness of the beads was measured by a Buehler microhardness tester using a load of $200 \mathrm{~g}$ and a 12 second dwell time. The measurements were performed at the center of the bead at a $100 \mu \mathrm{m}$ interval from the top of the bead, through the dilution and HAZ, and into the substrate material. Two measurements were performed at a $250-\mu \mathrm{m}$ distance, from each side of the first indentation.

To measure the stress introduced to the beads, a Proto X-ray diffraction system (Lab 002/LXRD 06024) was used for the first two samples presented in Table 2. Six points were taken along the centreline of the bead in where 0 is located at the top of the bead. The measurements were calculated through the bead, the dilution zone, the heat-affected zone, and the substrate material. Data for the as-clad and post heat treatment conditions were collected and used to calibrate the simulation model described in the next section [10].

\subsection{Simulation model}

The laser cladding simulation was performed by the coupled thermal-metallurgical-mechanical analysis with the FE software, SYSWELD. The results of the thermal analysis were used as an input of the mechanical and metallurgical analysis. In the FE model, the heat source was defined, and the boundary conditions were applied to the heat equation. The solver in the SYSWELD software solves a system of differential equations using a generalized trapeze method. The thermal and mechanical properties of AISI 1018 steel alloy were entered as an input in the FE software. The material chemical composition is shown in Table 2. A threedimensional moving heat source was applied in the 3D FE-model. The thermal properties such as the thermal conductivity, specific heat, and coefficient of thermal expansion and mechanical properties of the material such as Young's modulus, Poison's ratio, yield strength, and strainhardening curves are depicted in figure 5 [10]. 


\begin{tabular}{l|c|c|c|c|c|c|c|c|c|c}
\hline \multicolumn{1}{c|}{ Elements } & $\mathrm{C} \%$ & $\mathrm{Si} \%$ & $\mathrm{Mn} \%$ & $\mathbf{P \%}$ & $\mathrm{S \%}$ & $\mathrm{Cr} \%$ & $\mathrm{Ni} \%$ & $\mathrm{Cu} \%$ & $\mathrm{Sn} \%$ & $\mathrm{Co} \%$ \\
\hline AISI 1018 & 0.18 & 0.19 & 0.81 & 0.012 & 0.033 & 0.1 & 0.14 & 0.23 & 0.01 & - \\
\hline AISI 420 & 0.23 & 0.5 & 1.5 & 0.04 & 0.03 & 12.6 & - & - & - & 0.02 \\
\hline
\end{tabular}

Table 2. Chemical composition of the substrate and cladding powder
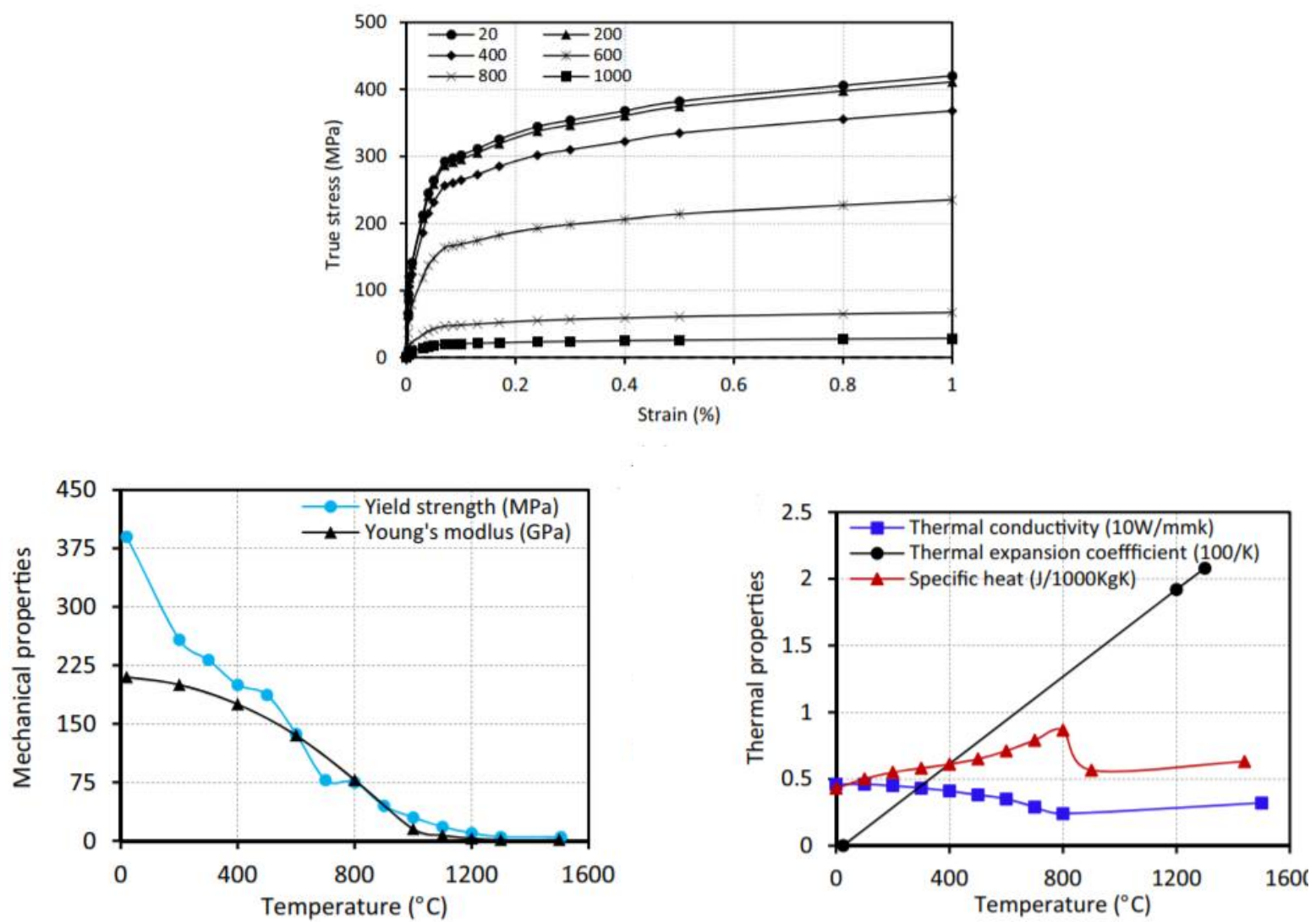

Figure 5. Stress- strain curve, temperature dependent mechanical properties and thermal properties of AISI 1018 steel [10]

The FE model was meshed using eight nodded hexahedron elements, four nodded surface elements and two nodded linear elements for the clad lines. The mesh for the single-track cladded specimens consists of 16,364 elements. The size of the uniform element of the substrate was $0.5 \times 0.5 \times 2 \mathrm{~mm}$ [10]. The input parameters to set up different iterations of the FE models of the single bead are the ones used in the experimental setup as shown in Table 1. It is noted that the measured bead geometry is used to create the mesh model for the FEA. Figure 6 demonstrates the geometry of the substrate used in FE model. 


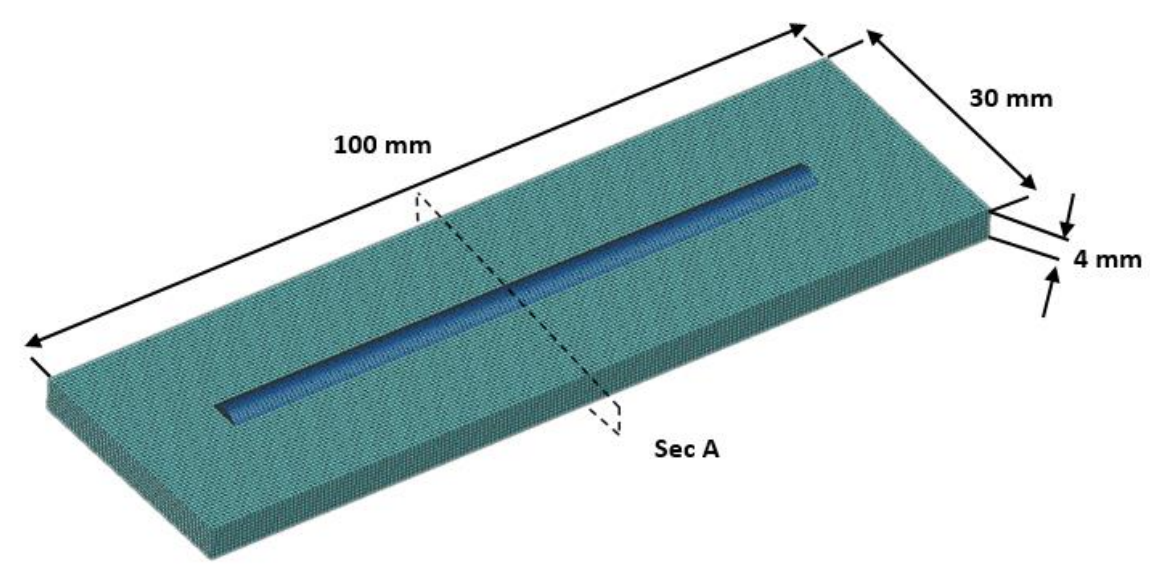

Figure 6. The FE model of the single bead clad and substrate

The residual stress in the middle section of the clad bead was measured through the depth of the bead for the single-track specimens. Figure 7(a) shows the experimental and simulation data, and Figure 7(b) illustrates the residual stress curves for all input configurations for the RSM design of experiments. Figure 8 shows the residual stress measurement through the depth of the single-track bead. The residual stress changes from tensile to compressive form top surface of the bead to the substrate through the depth and again tensile. 



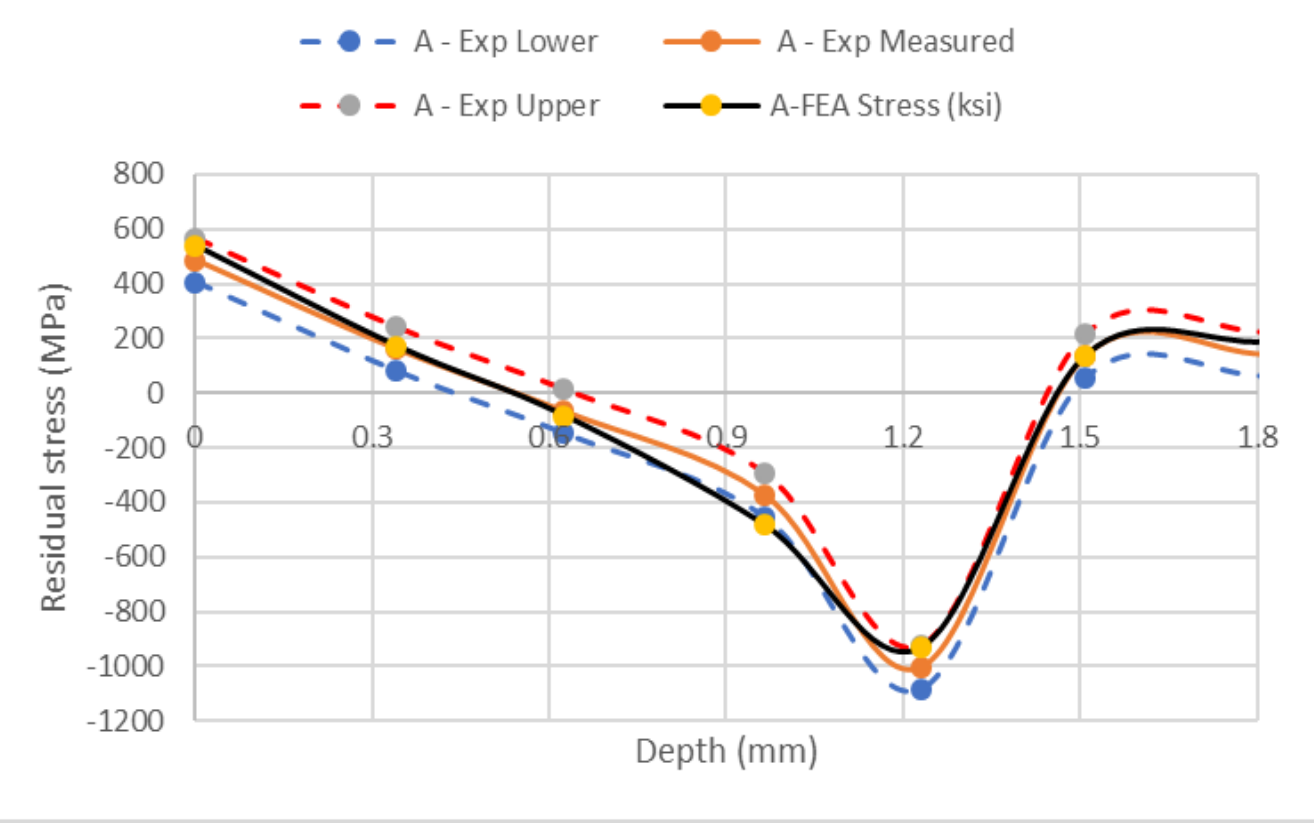

(a)

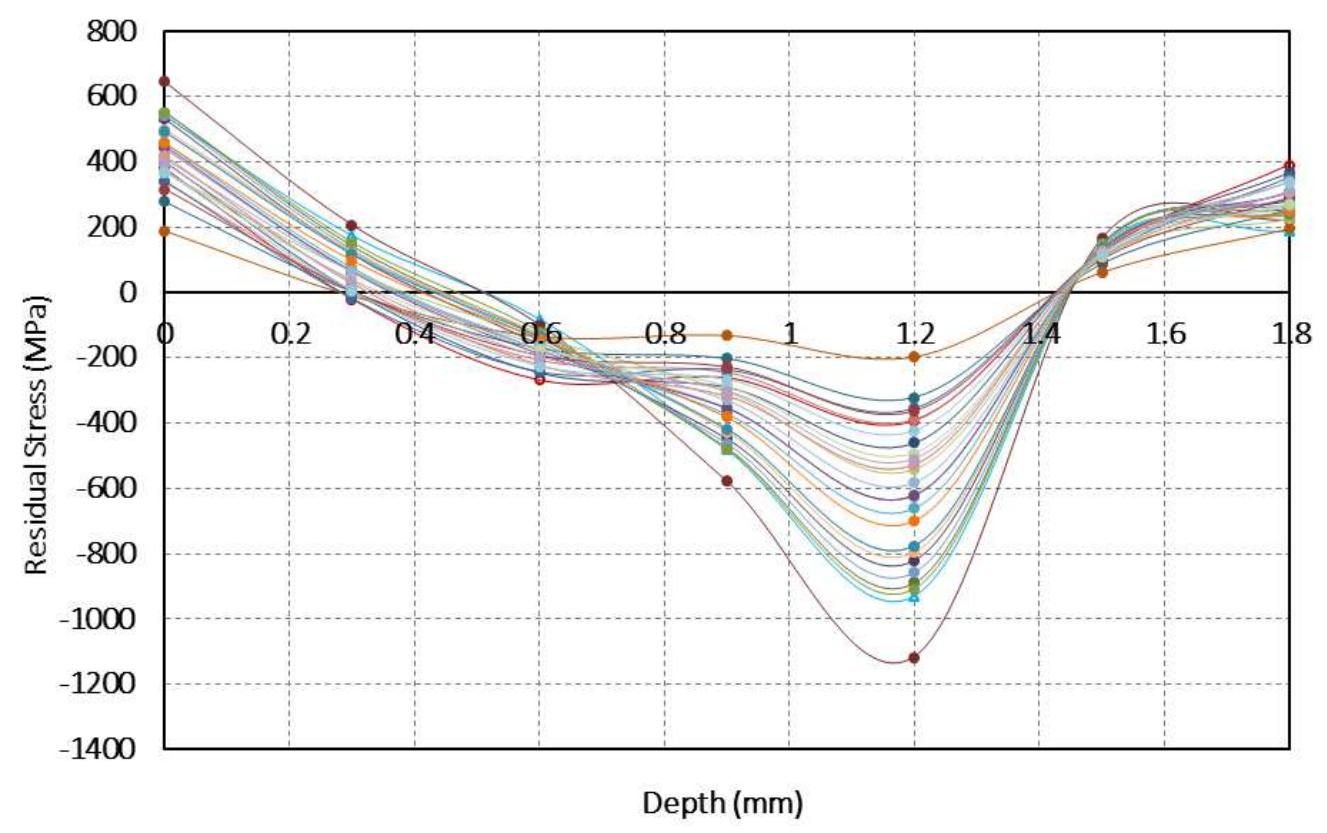

Figure 7. (a) Residual stress data, and simulation results [13] (b) residual stress simulation data for all experimental input variants 


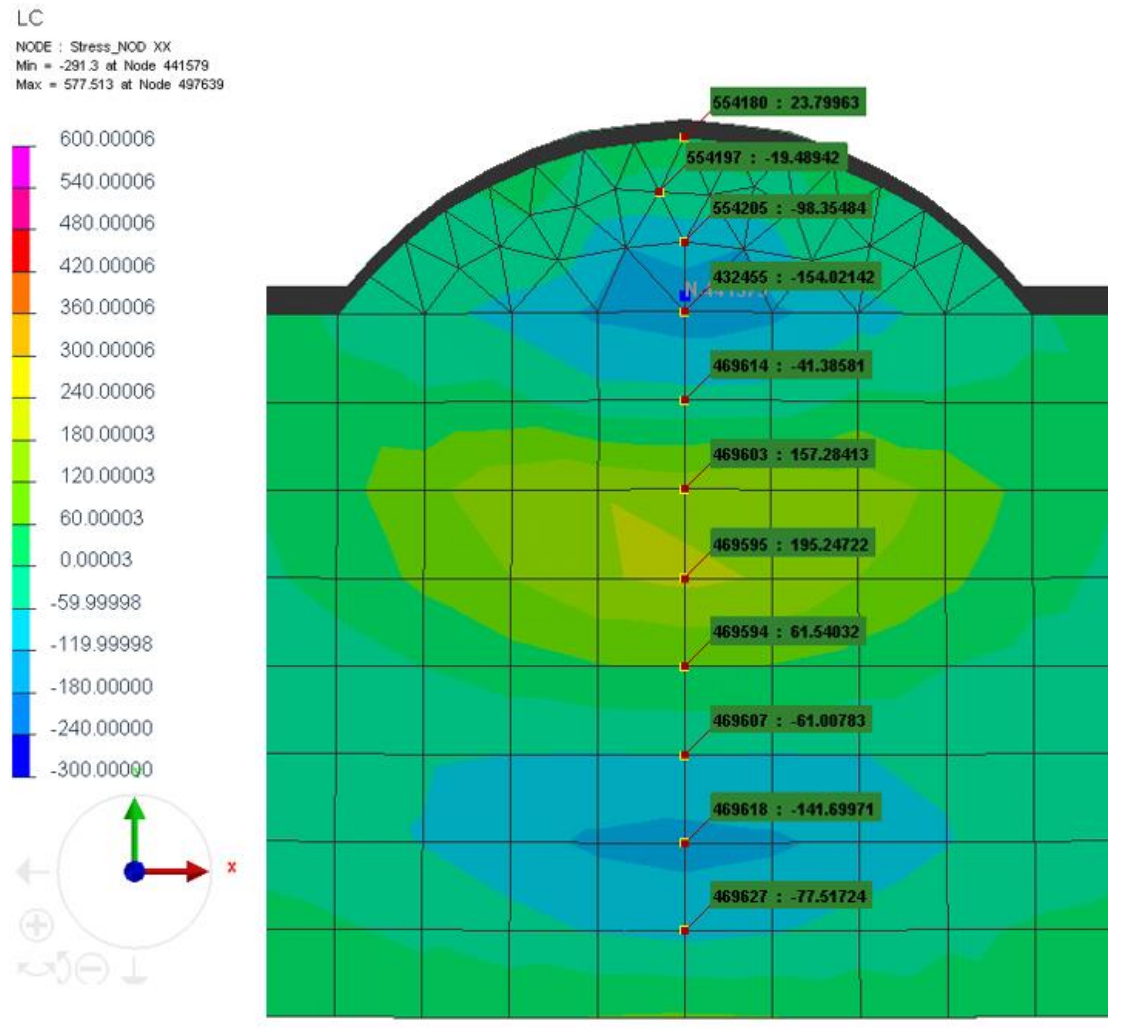

Figure 8. The measurement of residual stress in the FE model of the single bead clad and the substrate

Hardness correlates to yield strength, and can be utilized to characterization. The Vickers microhardness was measured experimentally using a Buehler microhardness tester. A load of $200 \mathrm{~g}$ and loading time $12 \mathrm{~s}$ were applied vertically in the centre of the bead keeping $150-\mu \mathrm{m}$ distance from each other form the bead surface through the substrate [10]. Figure 9 shows the hardness measurement through the depth of the single-track bead specimen. The hardness decreases moving from the top surface of bead through the depth to the substrate. 

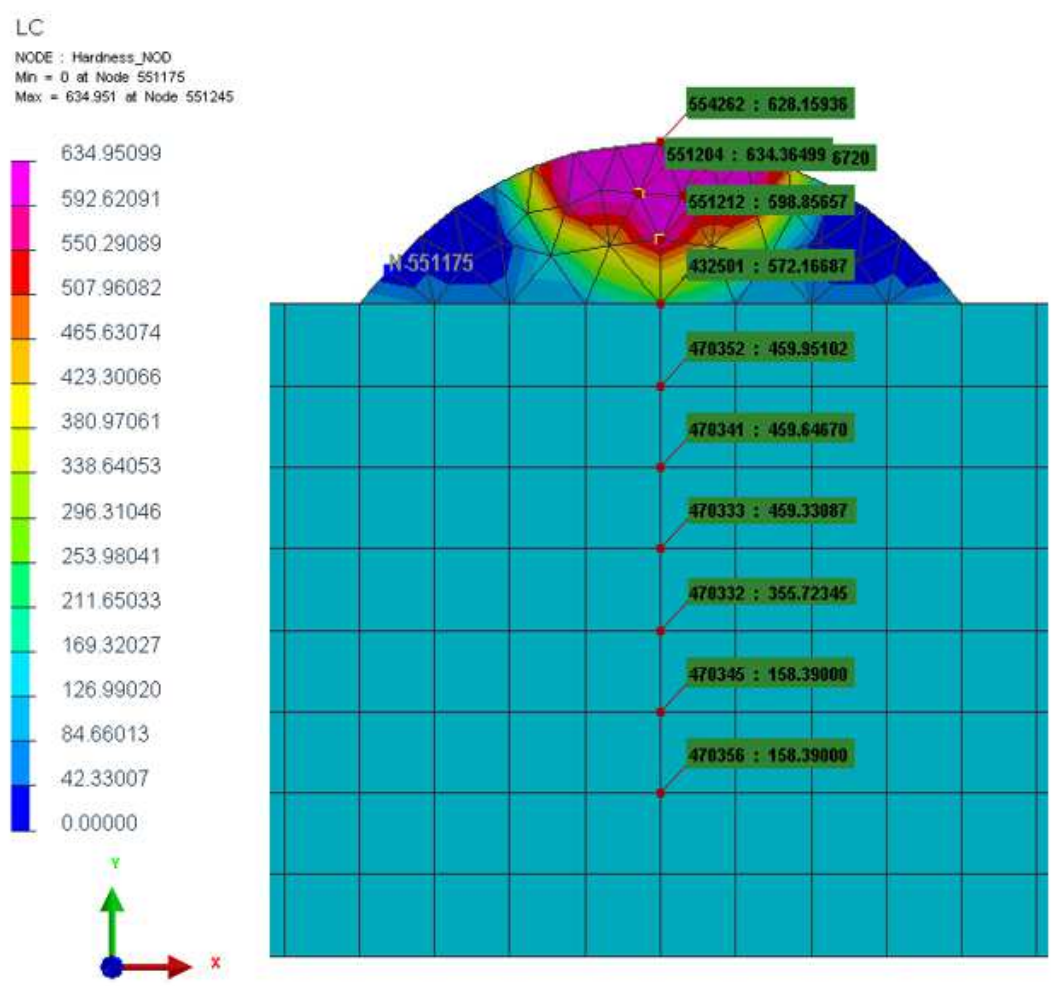

Figure 9. The measurement of hardness in the FE model of the single bead clad and the substrate

\section{Development of Mathematical Models}

Artificial neural networks (ANNs), like the biological neural network, contains neurons and activators to learn from supervised data. Among the various methods that have been proposed for the artificial neural networks, the feed-forward back-propagation method is well-suited to physical applications. This network normalizes the input domain, assigns the weights to the inputs, and sends the sum of the inputs with their associated weights to the next layer neurons. The weight assigned to the input or neurons represents the importance of that input or neuron. The activator then maps the calculated values for each neuron to the interval between minus one and one. The outputs of the neurons with weights are sent finally to the last layer. However, this method uses bias to reduce disturbance and control computation. The calculations start with the initial guess of weights and bias. They are modified by optimizing the gradient of these 
guesses. The objective function, which is the difference of the actual output values from the predicted output, is optimized.

As a result, the neural network can specify an analytical mathematical model for correlating inputs to physical outputs. However, this method fails to detect constructive physical parameters in predicting outputs and provides the model solely based on what the user defines as data. However, once the inputs are introduced, the neural network will be able to correlate the input to determine the outputs. Also, there is no unique criterion for determining the number of layers and neurons for the best prediction.

The Adaptive Neuro Fuzzy inference system was also explored to predict residual stress and hardness in 1D, 2D and 3D domains for the single laser clad bead. The ANFIS model combines the best features of a Neural Network System and a Fuzzy system. The structure of an ANFIS model is demonstrated in Figure 10. An ANFIS is used to map input characteristics to input membership functions, input membership function to a set of TSKtype fuzzy if-then rules, rules to a set of output characteristics, output characteristics to output membership functions, and the output membership function to a single-valued output or a decision associated with the output [22][23][24]. In ANFIS model the exact same input parameters which were used as an input to train the ANNs model are employed.

At the computational level, ANFIS can be regarded as a flexible mathematical structure that can approximate a large class of complex nonlinear systems to a desired degree of accuracy [23][24].

To clarify, assume that the fuzzy inference system has two inputs $\mathrm{x}$ and $\mathrm{y}$, and one output $\mathrm{f}$. For the first order Sugeno fuzzy model, a single fuzzy if-then rule assumes the form:

Rule number1 if $\mathrm{x}$ is $\mathrm{A} 1$ and $\mathrm{y}$ is $\mathrm{B} 1$, then:

$$
f_{1}=p_{1} x+q_{1} y+r_{1}
$$


Rule number 2 if $\mathrm{x}$ is $\mathrm{A} 2$ and $\mathrm{y}$ is $\mathrm{B} 2$, then:

$$
f_{1}=p_{2} x+q_{2} y+r_{2}
$$

Where $p_{i}, q_{i}$ and $r_{i}$, are linear output parameters:

- Layer 1: Every node in this layer contains membership functions described by the triangular function [25].

$$
\mu_{A}(x)=\frac{1}{1+\left|\frac{x-c_{i}}{a_{i}}\right|^{2 b_{i}}}
$$

where $a_{i}, b_{i}$ and $c_{i}$ are referred to premise parameters.

- Layer 2: Every node in this layer is a fixed node and calculates the firing strength of a rule multiplication.

- Layer 3: Every node in this layer calculates the weight, which is normalized. The outputs of this layer are called normalized firing strengths.

- Layer 4: This layer output is a linear combination of the inputs multiplied by the normalized firing strength.

- Layer 5: This layer is the summation of the layer 4 outputs.

The adjustment of the modifiable parameters is a two-step process. First, the consequent parameters are identified by the least. square estimation, then the premise parameters are updated by the gradient descent [23][24]. 


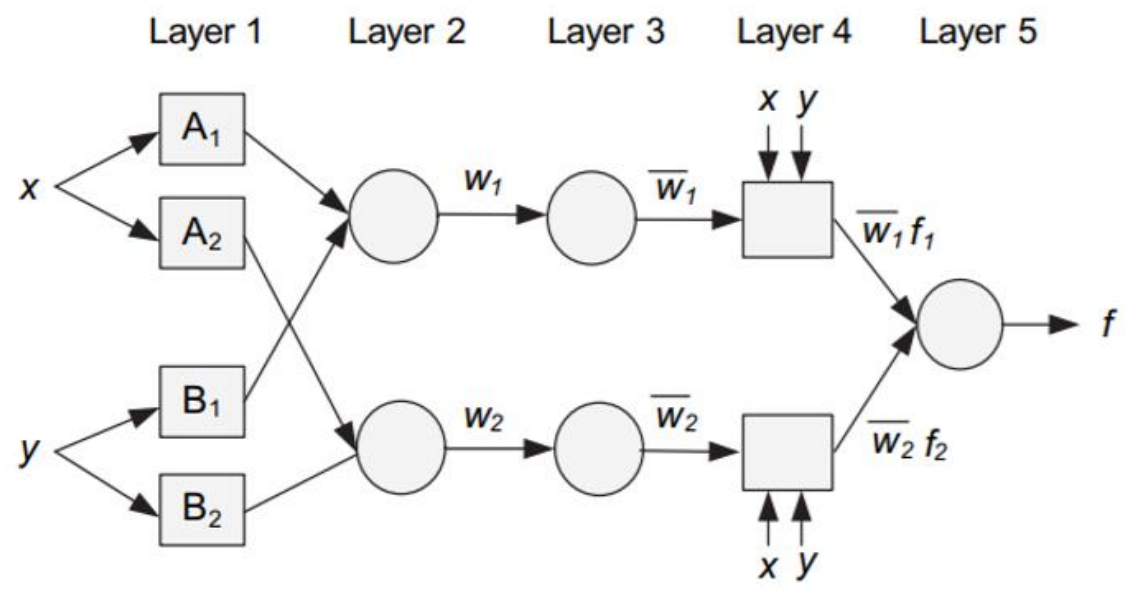

Figure 10. The ANFIS model structure based on Takagi-Sugeno [18]

The mean relative error (MRE) is defined by the following formula, equation (4), to be used for performance comparison between ANFIS and ANN model [24].

$$
M R E=\frac{1}{N} \times \sum_{i=1}^{N}\left|\frac{\left.X_{i}(\exp )-X_{i}(\text { pred })\right)}{X_{i}(\exp )}\right|
$$

In this equation $X(\exp )$ shows the actual data and $X($ pred $)$ stands for the predicted data by mathematical models. $\mathrm{N}$ is the number of data.

\subsection{Mathematical models in the 1D domain}

To calculate the geometry, hardness, and residual stress in a 1D domain, the following forward network architecture (MLP Network) was developed for a single pass DED Process. The input parameters including the powder feed rate (X1), laser power (X2), focal length of the lens (X3), laser speed (X4), and the contact tip to work piece distance (X5) were connected to the hidden layer.

For the hidden layer, a Tan Sigmoid activation function (40 Neurons) and for the output layer, a linear activation function (7 Neurons) is developed. The predicted outputs were width, height, penetration, dilution, hardness, tensile residual stress and compressive residual stress. Here a 
70-15-15 (Training-Testing-Validation) division of data is used to obtain the best prediction results. A schematic view of the proposed artificial neural network (ANN) has been shown in Figure 11.

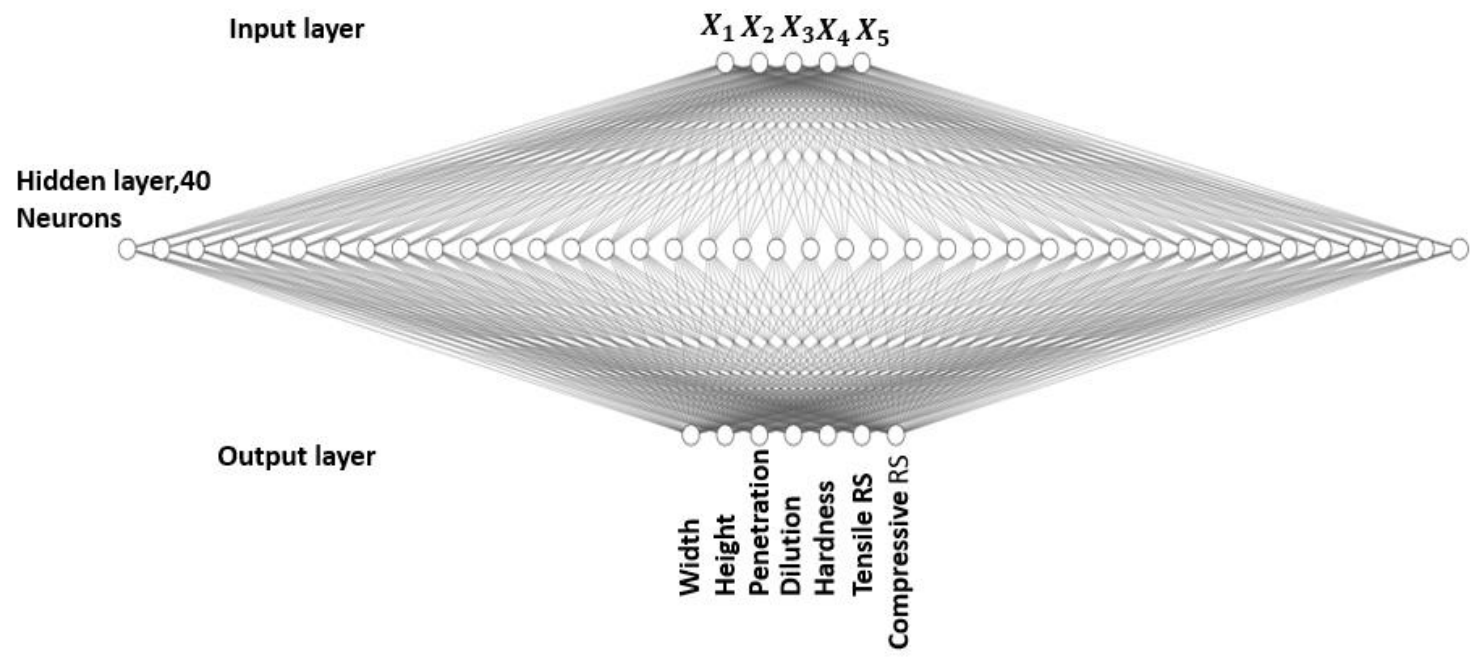

Figure 11. ANN Structure for the 1D domain. Network inputs include PW, FR, FL, LS and CTWD. The outputs of the network are W, H, P, D, HRDNS, TRS and CRS

\subsection{Mathematical Models in 2D domain}

In this application, the model is trained using the input data from the finite element models and validated by comparing its output with the experimental data and FEA results. As with the 1D model, the input layer consisting five input parameters, the powder feed rate (X1), laser power (X2), focal length of the lens (X3), laser speed (X4), and the contact tip to work piece distance (X5), is connected to three hidden layers. The hidden neurons are then connected to the output layer. The back-propagation algorithm used is the Levenberg-Marquardt algorithm as the training function; the mean relative error (MRE) was considered as a performance criterion during the training of the feed-forward back-propagation network. The data set used to train the model was divided into training set ( $85 \%$ of the data) and the test and verification set (15\% of the data). Figure 12 shows the structure of the neural network model. The model with a 20- 
10-10 architecture was selected as the best model performance for predicting the residual stress and hardness, with a three hidden layers model network.

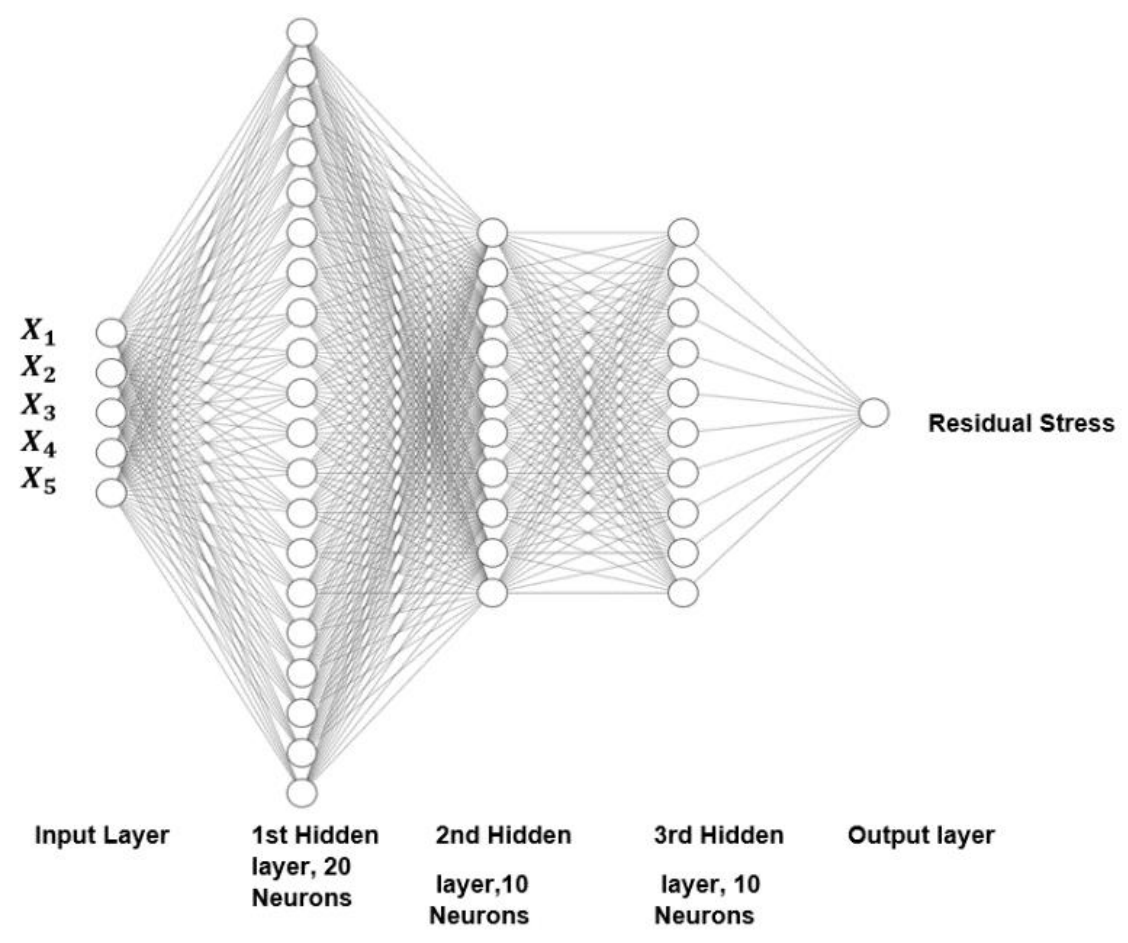

Figure 12. Artificial neural network architecture for selected input and output [15]

\subsection{Mathematical models in the 3D domain}

It has been previously discussed how machine learning methods can be used to predict the geometrical and mechanical characteristics of the metal for the 1D and 2D domains. In parts that have been exposed to multiple heating and cooling cycles, thermal residual stresses result in completely non-uniform stresses. Any symmetry assumptions could therefore lead to unrealistic results for residual stresses and hardness. As a result, residual stresses and hardness change locally and in order to avoid failure, the entire domain of a part should be simulated before being manufactured to determine whether the part is in the safe domain and there is no point exceeding the yield strength. 
The purpose of this section is to explain how to analyze residual stresses and hardness at every single point in a part using ANN and ANFIS models. A comparison is made between ANN and ANFIS models with regards to residual stress and hardness prediction.

The feed-forward back-propagation algorithm is used in the current neural network. The input and output data, as well as the general architecture of the feed-forward back-propagation neural network is shown in Figure 13. The $\mathrm{x}, \mathrm{y}$, and $\mathrm{z}$ coordinates are three geometric features, and the laser speed and power are two process parameters for determining residual stress. However, the structure of available data in this study allowed these variables to be selected and does not mean these are the only effective parameters to determine residual stresses.

For the data collection, a finite element three-dimensional simulation of one bead has been conducted using SYSWELD software. In that simulation, one bead in nine different sets of process parameters is modelled. This part contains 115000 finite element cells, and each cell is regarded as one sample data. Laser speed and laser power are two variables. Each cell gets three different laser powers of $1.5,1.8$, and $2 \mathrm{KW}$, with laser speeds of 8,10 , and $12 \mathrm{~mm} / \mathrm{sec}$, which results in nine distinct simulation results. As a result, the maximum number of samples is $115000 \times 9=1035000$. However, 115000 data is selected from the heat affected zone to train the ANNs and ANFIS models. 
Input layer

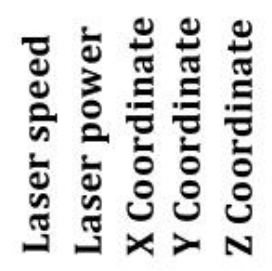

$1^{\text {st }}$ hidden

layer,20 Neurons

2nd hidden

layer,20 Neurons

Output layer

Residual Stress

Figure 13. General architecture of the neural network

After a systematic assessment to determine the number of layers and neurons, it was found that for this data structure, a neural network with one hidden layer is able to predict the residual stress with good performance. A summary of the type of network, the number of samples and features investigated for the 3D model are presented in Table 3.

\begin{tabular}{|l|l|}
\hline ANN type & Feed-Forward \\
\hline Number of layers & 1 \\
\hline Number of neurons & 9 \\
\hline Activation functions & TanSig \\
\hline Number of data Samples & 115000 \\
\hline Number of input features & 5 \\
\hline Number of output features & 1 \\
\hline
\end{tabular}

Table 3. Neural network information for 3D data 


\section{Results and Discussion}

\section{1 $1 D$ modelling results}

The 1D model performance results are shown in Figure 14, where a regression plot constructed between the target and the network output values is shown. The overall fitness of the network is equal to 0.976 which represents a very good fit. This is aligned with the performance characteristics when considering geometric characteristics only [5]. In Figure 15, the actual value and the network output for the bead hardness are plotted. The network has been consistent with following the trends in the data. However, in some samples, there are minor differences between the actual data and the network output. The residual error is mostly below $50 \mathrm{RCK} \mathrm{C}$ as demonstrated in Figure 16. Overall, the network has been able to provide a good correlation to the experimental hardness data, and more data should improve the results. 

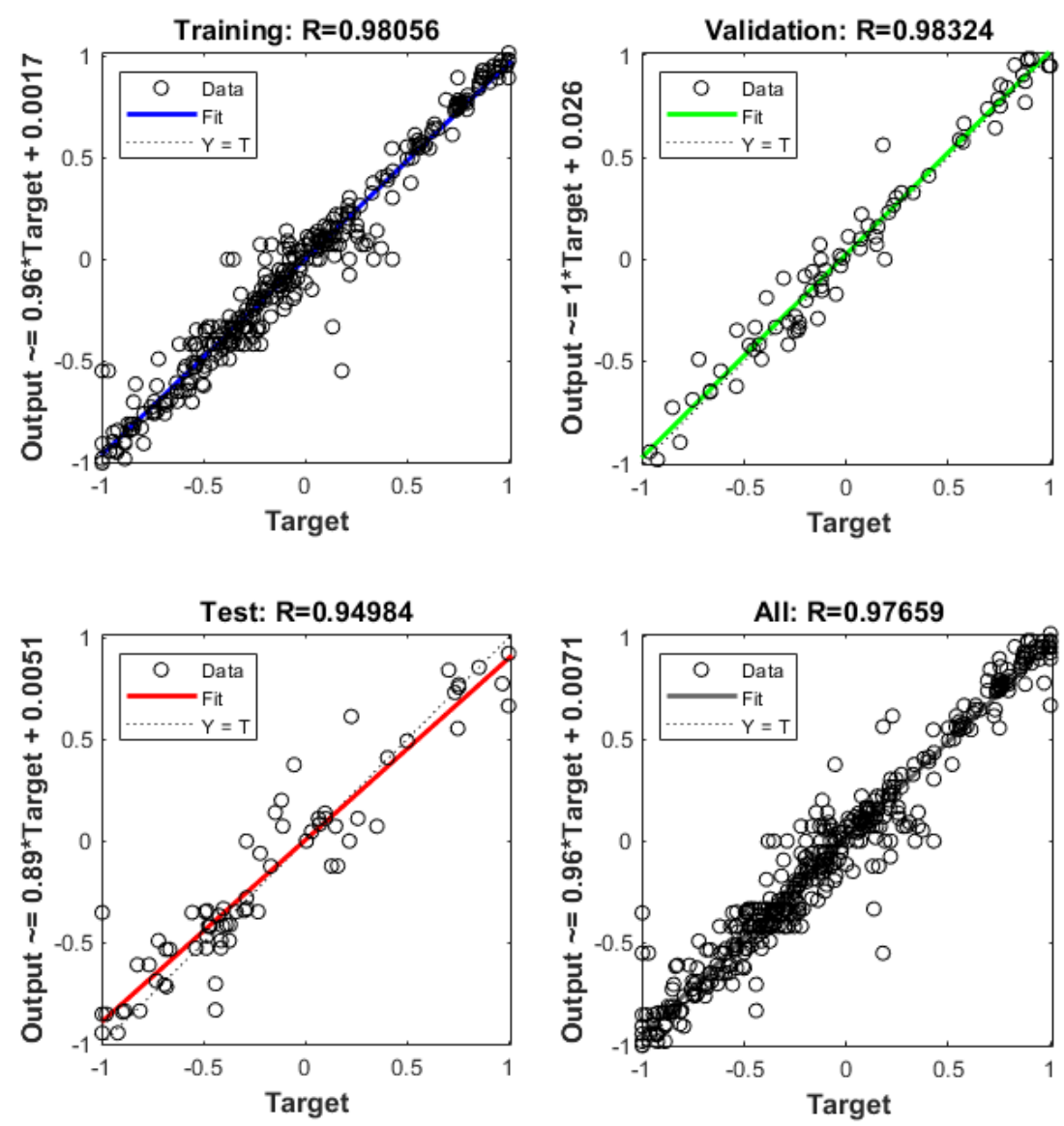

Figure 14. Regression plot of the developed neural network in 1D domain

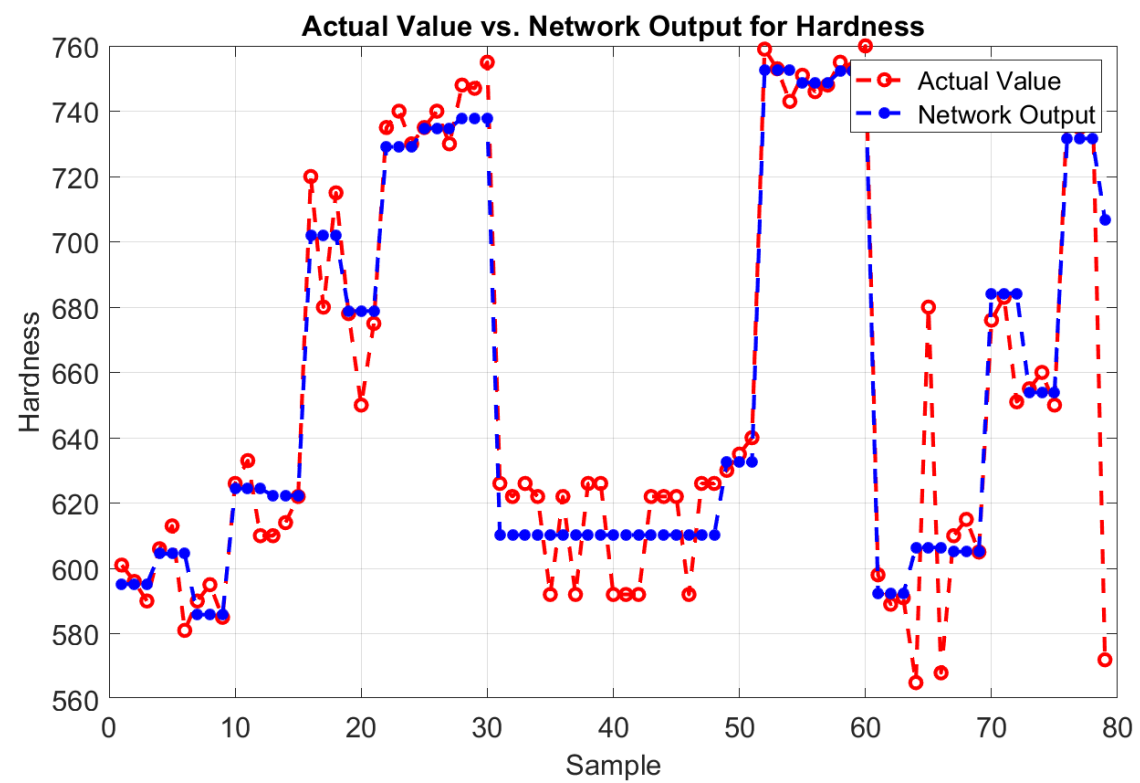

Figure 15. Actual value vs the network value of the bead hardness (RCK C) 


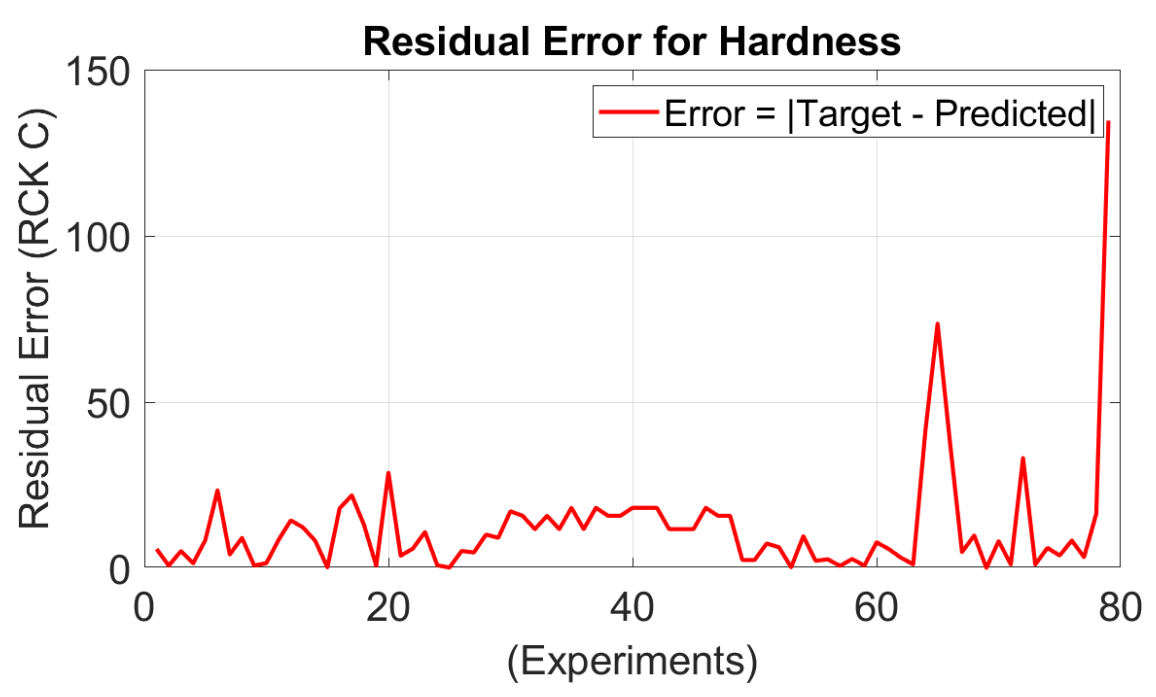

Figure 16. Residual error in bead hardness predictions

As shown in Figure17, the ANN model has been able to generate relatively accurate predictions for the residual stress. In Figure 18, the residual error for the residual stress has been plotted. The absolute error is below $5 \mathrm{ksi}$ between the actual and the predicted stress values for most points. 


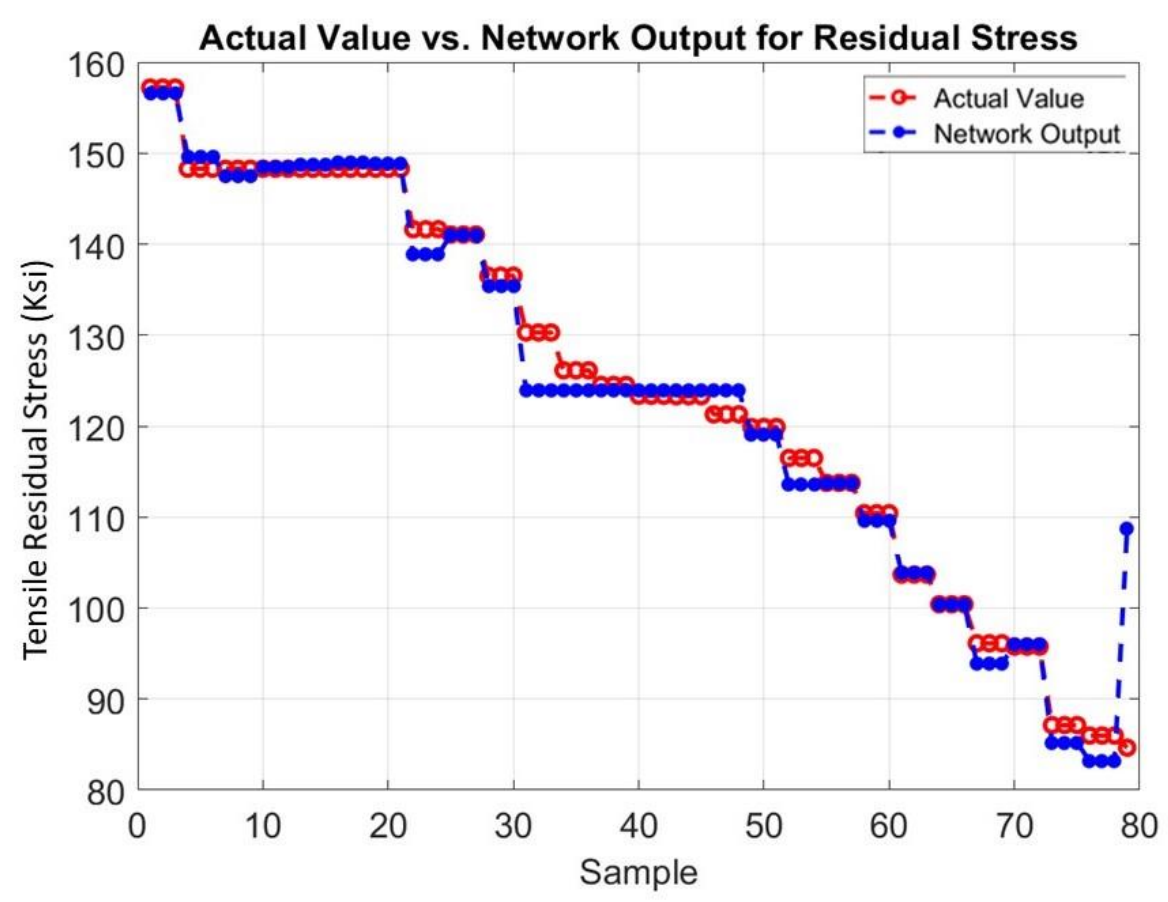

Figure 17. Actual value vs network output for tensile residual stress (Ksi)

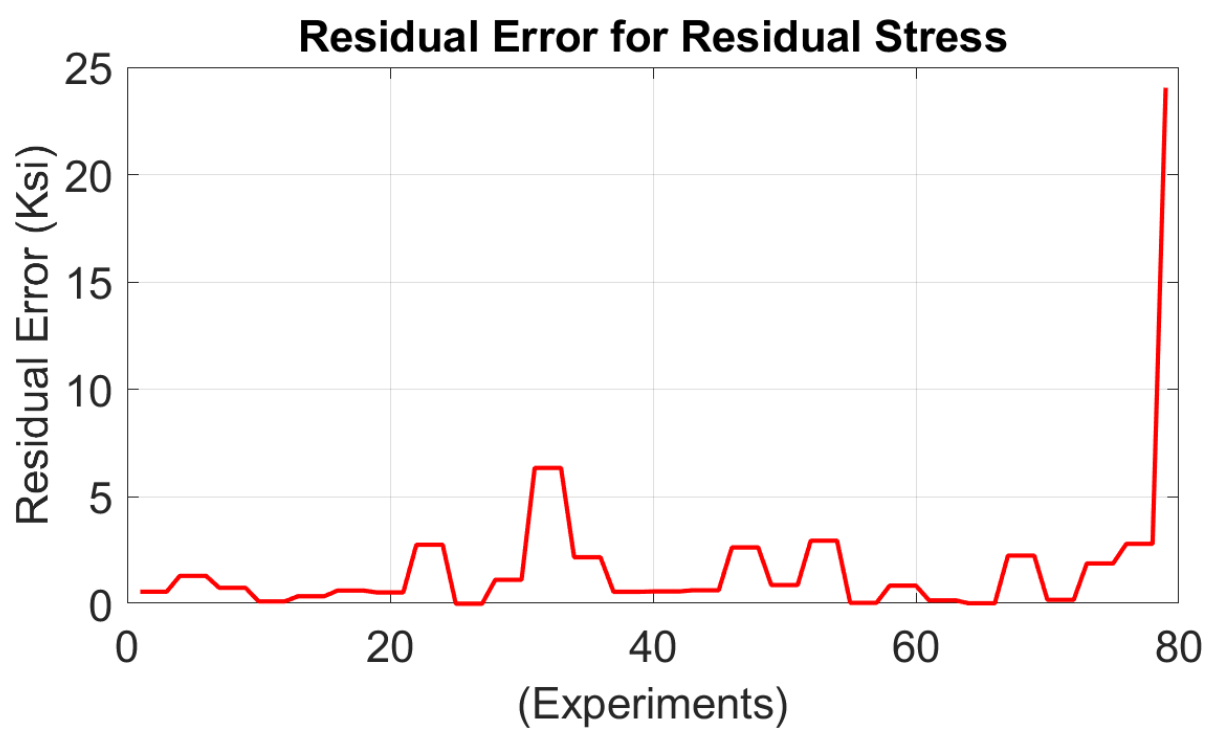

Figure 18. Residual error for maximum tensile residual stress predictions

The ANFIS model was used to predict the residual stress and the hardness as a multi input single output model. The performance of both ANFIS and ANN model were compared by calculating the MRE in Tables 4 and 5. The results show that the performance of ANFIS model to predict residual stress and hardness is better than the neural network. Figure 19 the residual 
stress-laser speed diagram being predicted by ANFIS, ANN and the actual data. Figure 20 shows the microhardness-laser speed diagram being predicted by ANFIS, ANN and the actual data.

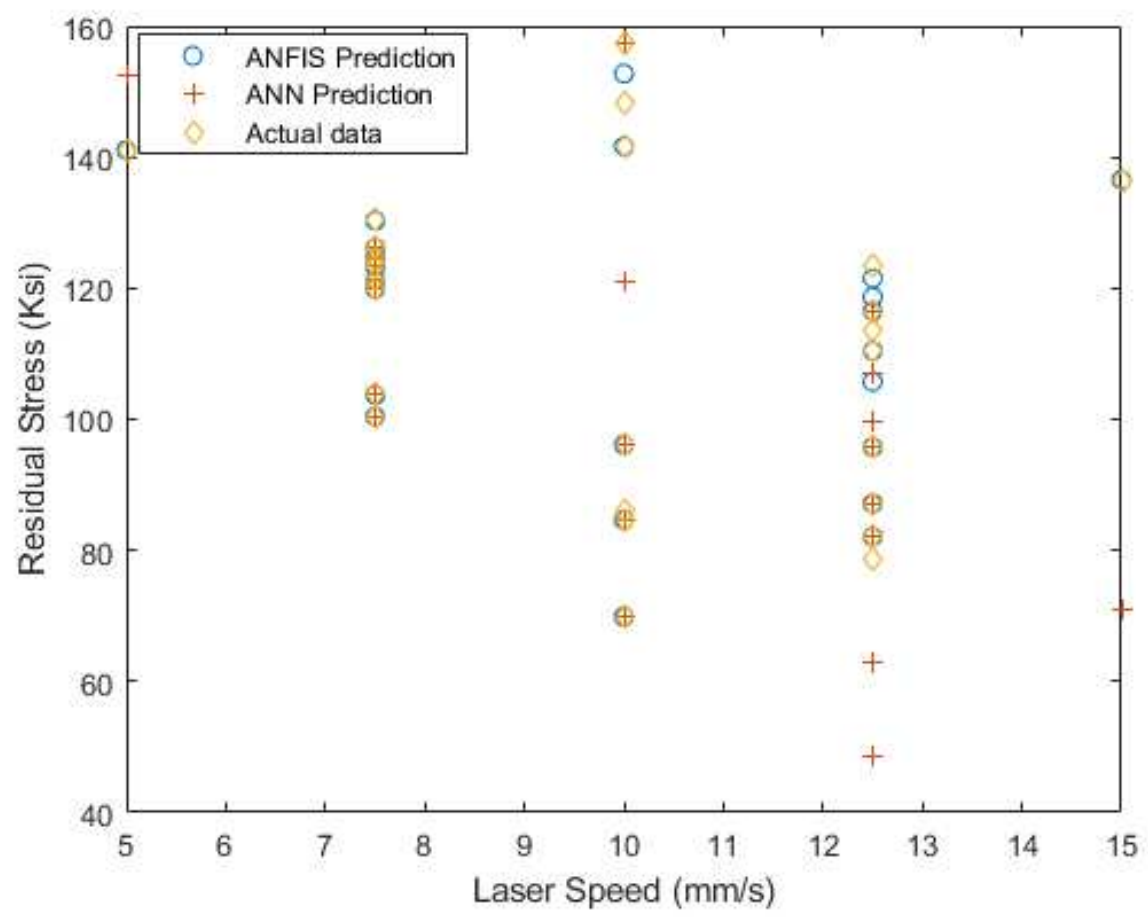

Figure 19. Comparison of residual stress results predicted by ANFIS, ANN and actual data

The performance of both ANFIS and ANN models were compared using the MRE (Table 6). The calculated MRE indicates that the ANFIS method has superior performance.

\begin{tabular}{|c|c|}
\hline Network & MRE \\
\hline ANFIS & 0.05 \\
\hline ANN & 0.08 \\
\hline
\end{tabular}

Table 4. Comparison between ANFIS and ANN models' performance, predicting the residual stress using Mean Relative Error 


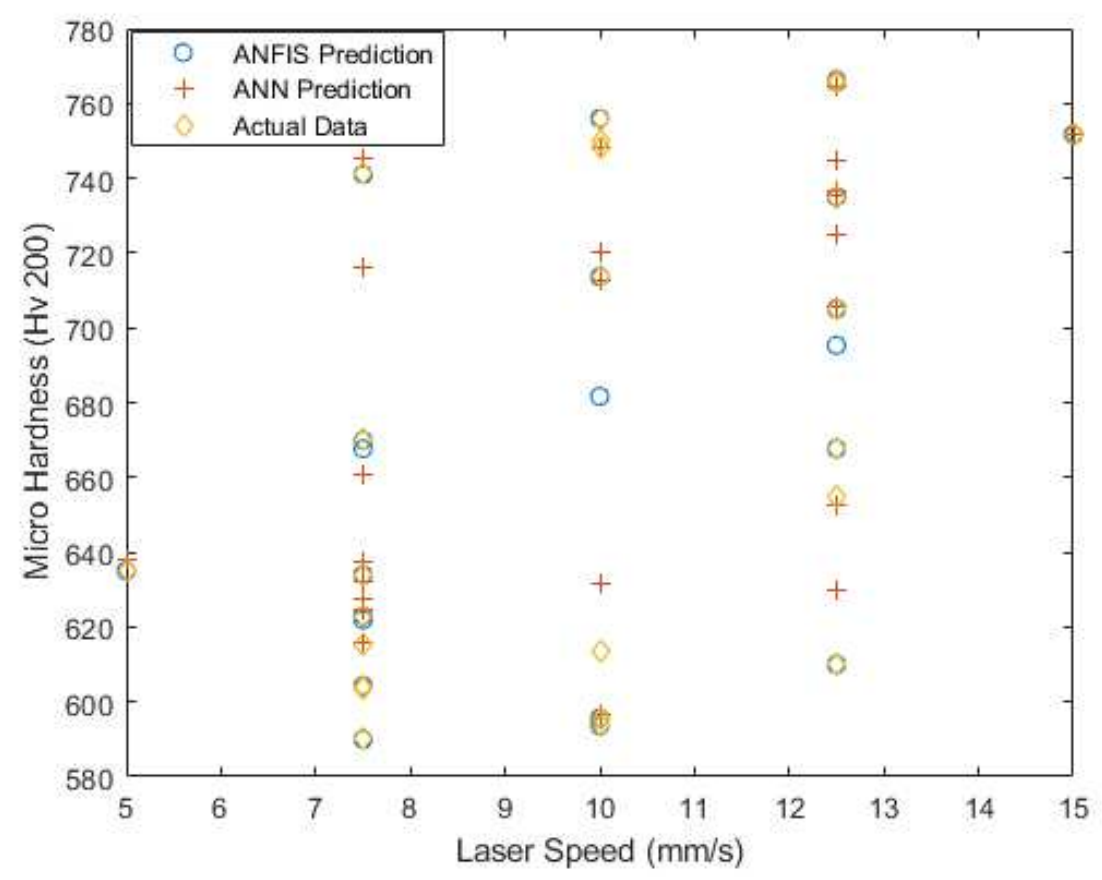

Figure 20. Comparison of Hardness results predicted by ANFIS, ANN and actual data

\begin{tabular}{|c|c|}
\hline Network & MRE \\
\hline ANFIS & 0.09 \\
\hline ANN & 0.1 \\
\hline
\end{tabular}

Table 5. Comparison between ANFIS and ANN models' performance, predicting the hardness using Mean Relative Error as a comparison

Although the maximum and minimum residual stresses could be predicted with a relatively high level of confidence, no location data is included. The 2D modelling illustrates this.

\section{$4.22 \mathrm{D}$ modelling results}

The 2D cross section ANN and ANFIS results are explored for 10 and 26 sample data sets for selected curves. The residual stress results are shown in Figure 21. The MRE is evaluated for the ANFIS model and ANN models (Table 6). 
(a)

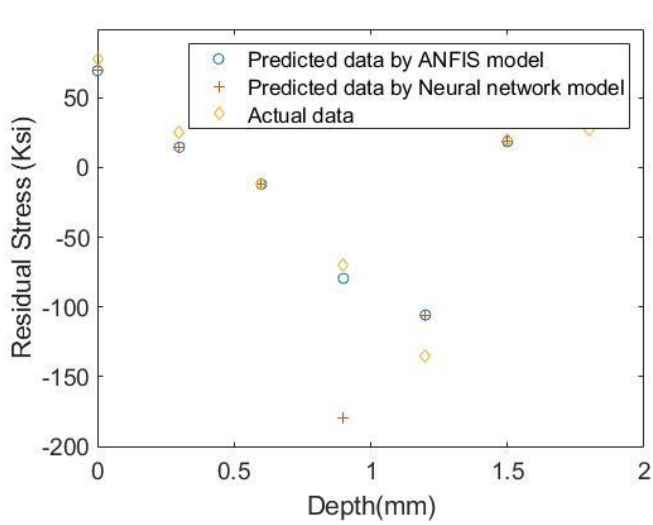

(b)

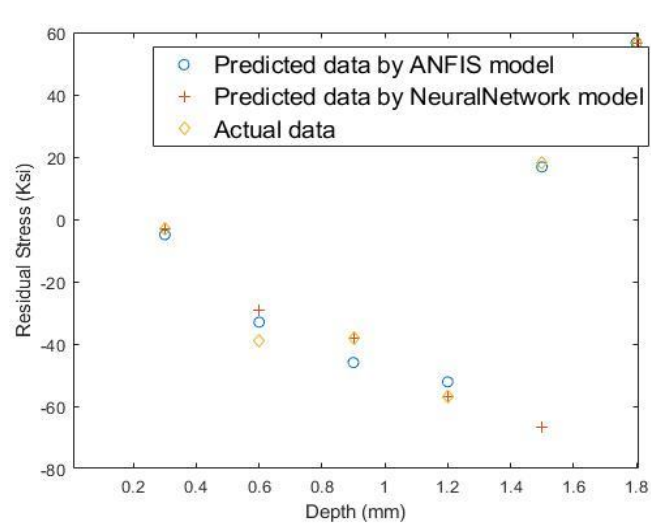

(c)

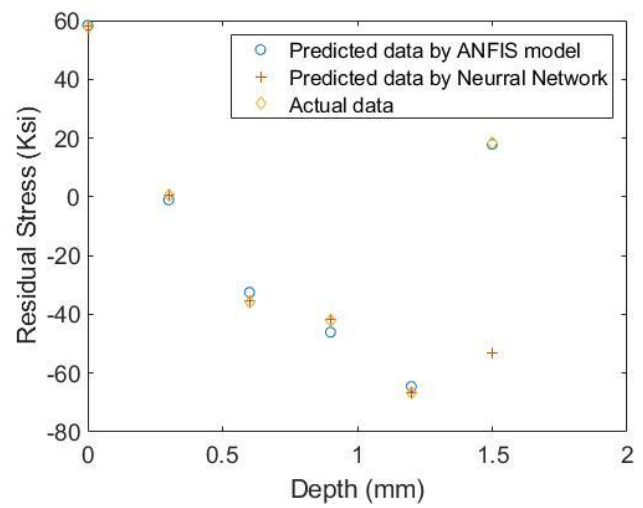

(aa)

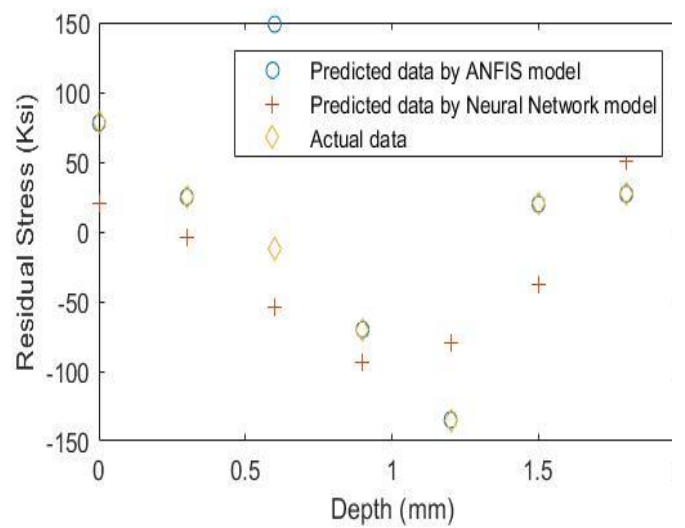

(bb)

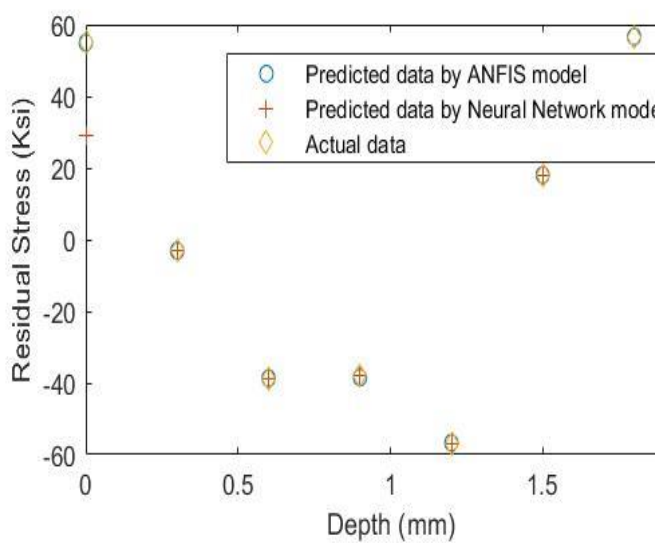

(cc)

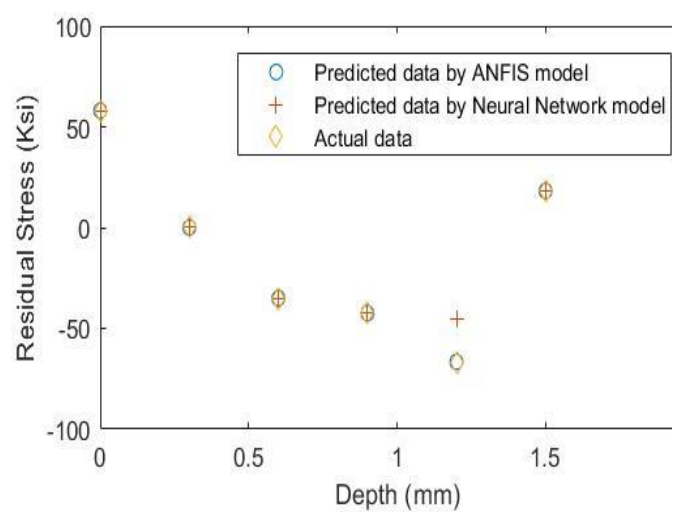

Figure 21. Residual stress results, 10 data samples for (a) data set A, (b) data set B, and (c) data set E. Residual stress results, 26 data samples for (aa) data set A, (bb) data set B, and (cc) data set $\mathrm{E}$

For both the 10 and 26 sample data sets, it was observed that the MRE for the ANFIS model is lower than those for the ANN results. It was observed that that ANFIS model converges in less time than the ANN model. The results predicted by these models agreed with the output of the finite element model and showed a good accuracy in prediction [26]. 
The regression plot is displayed to validate the network performance. The regression plot shows the network outputs with respect to targets for training, validation, and test sets.

\begin{tabular}{|c|c|c|}
\hline MRE & 10 Data set & 26 Data set \\
\hline ANFIS & 0.2 & 0.09 \\
\hline ANN & 0.3 & 0.1 \\
\hline
\end{tabular}

Table 6. Comparison between ANFIS and ANN models predicting the residual stress, using Mean Relative Error as a comparison for 10 and 26 data samples

The 10-sample data set (Figure 22) fits are not as good as the 26-sample data set as expected. For the 26-sample data set (Figure 23), the fit is reasonably good for all data sets, with the R values in each case $\mathrm{R}>0.91$.
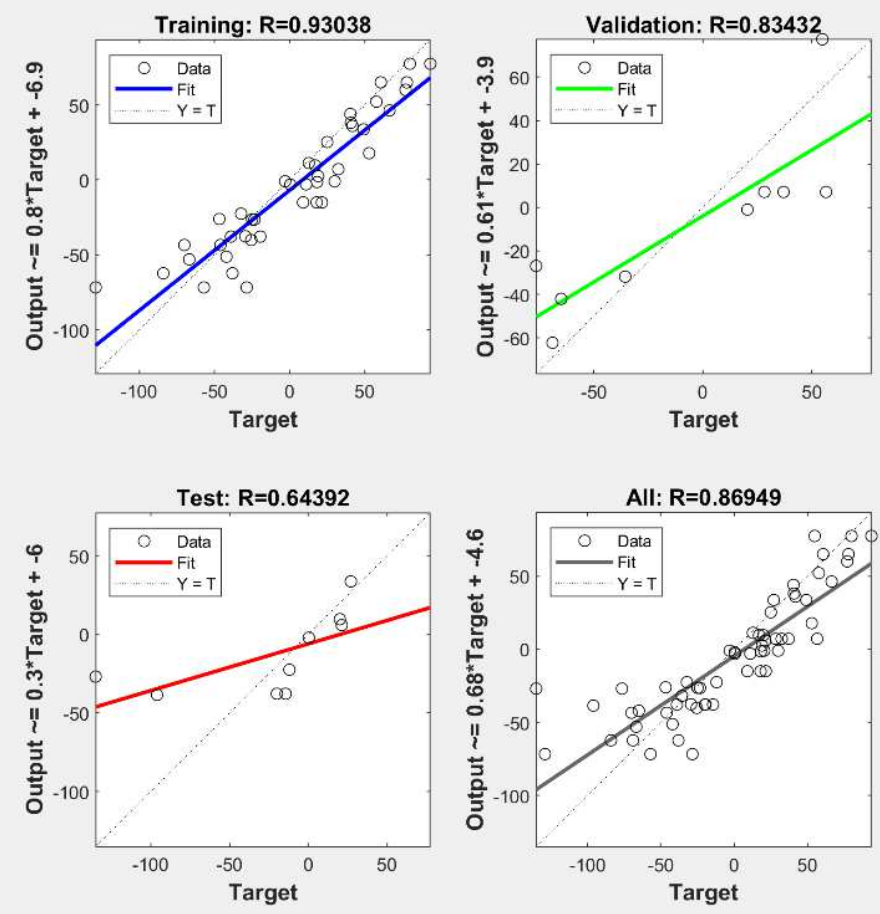

Figure 22. Regression plot using 10 data sets 

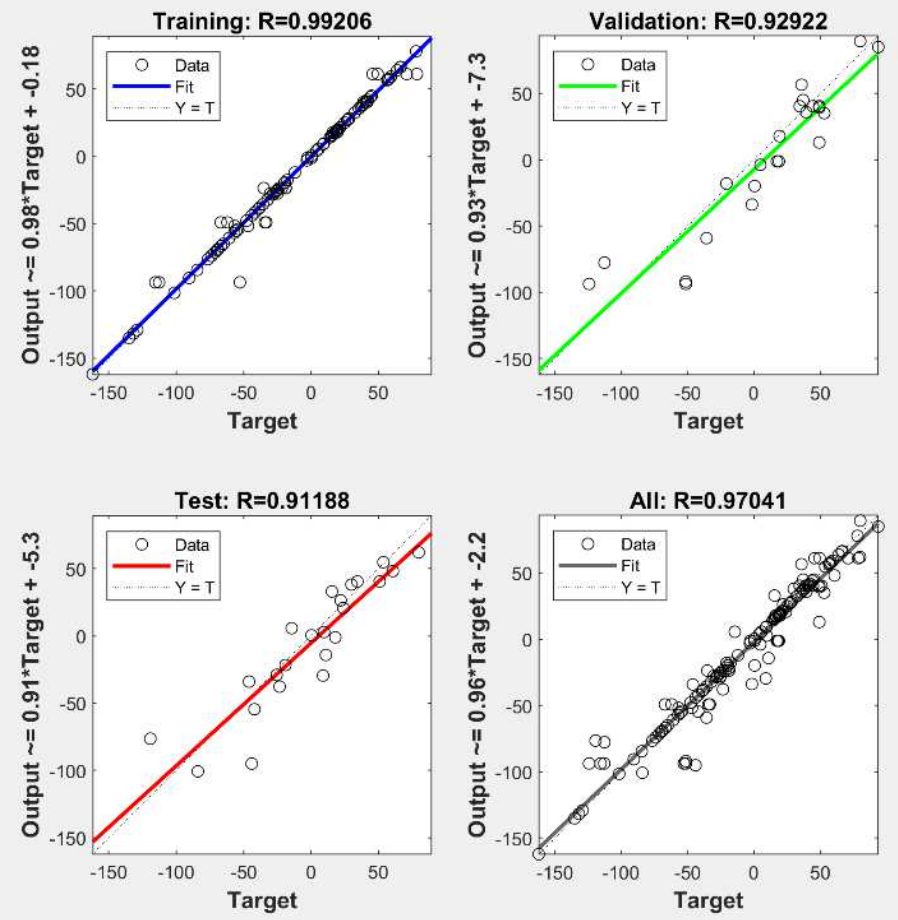

Figure 23. Regression plot using 26 data sets

The micro hardness predicted by ANFIS, ANN and the experimental data for the 2D domain are shown in Figure 24.

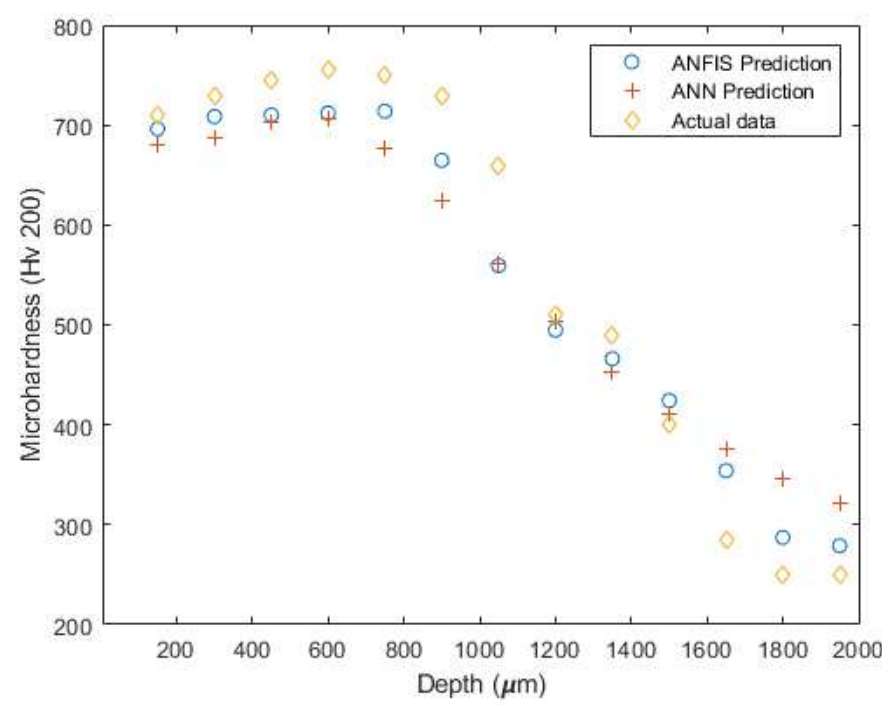

Figure 24. Comparison of hardness results predicted by ANFIS, ANN and actual data 
The MRE for the ANFIS and ANN models are summarized in Table 7. Less variation occurred for the hardness predictive modelling for the $2 \mathrm{D}$ domain, which is different than what is observed for the 1D domain MRE results. For both the residual stress and hardness results the calculated MRE indicates that the ANFIS model has superior performance.

\begin{tabular}{|c|c|}
\hline Network & MRE \\
\hline ANFIS & 0.08 \\
\hline ANN & 0.09 \\
\hline
\end{tabular}

Table 7. Comparison between ANFIS and ANN models' performance, predicting the hardness using Mean Relative Error as a comparison

\section{$4.33 D$ modelling results}

This section discusses residual stress and hardness prediction in the $3 \mathrm{D}$ domain followed by a sensitivity analysis of these two approaches. Feed-forward back-propagation with one hidden layer is used for the ANN and two membership functions are used to achieve acceptable predictions when using ANFIS. However, in the 3D domain there is a large data set with several near-zero values of residual stresses. Therefore, using the MRE for data with near-zero values may result in a high error. For example, a 5 Ksi stress prediction may occur for the actual value of $1 \mathrm{Ksi}$, which results in a relative MRE of $400 \%$. However, the prediction of $5 \mathrm{Ksi}$ for a 1 Ksi data value is extremely good where the maximum residual stress is $450 \mathrm{Ksi}$ in the part. Therefore, for the purpose of calculating the MRE, we selected residual stress data whose absolute value exceeded $100 \mathrm{Ksi}$ to avoid misinterpretation of the models' performance. Due to the fact that the hardness values are far from zero, all the data points are used to calculate the MRE.

First, the effects of the number of neurons on the performance of the neural network is examined in transverse residual stress prediction (Figures 25 and 26). 


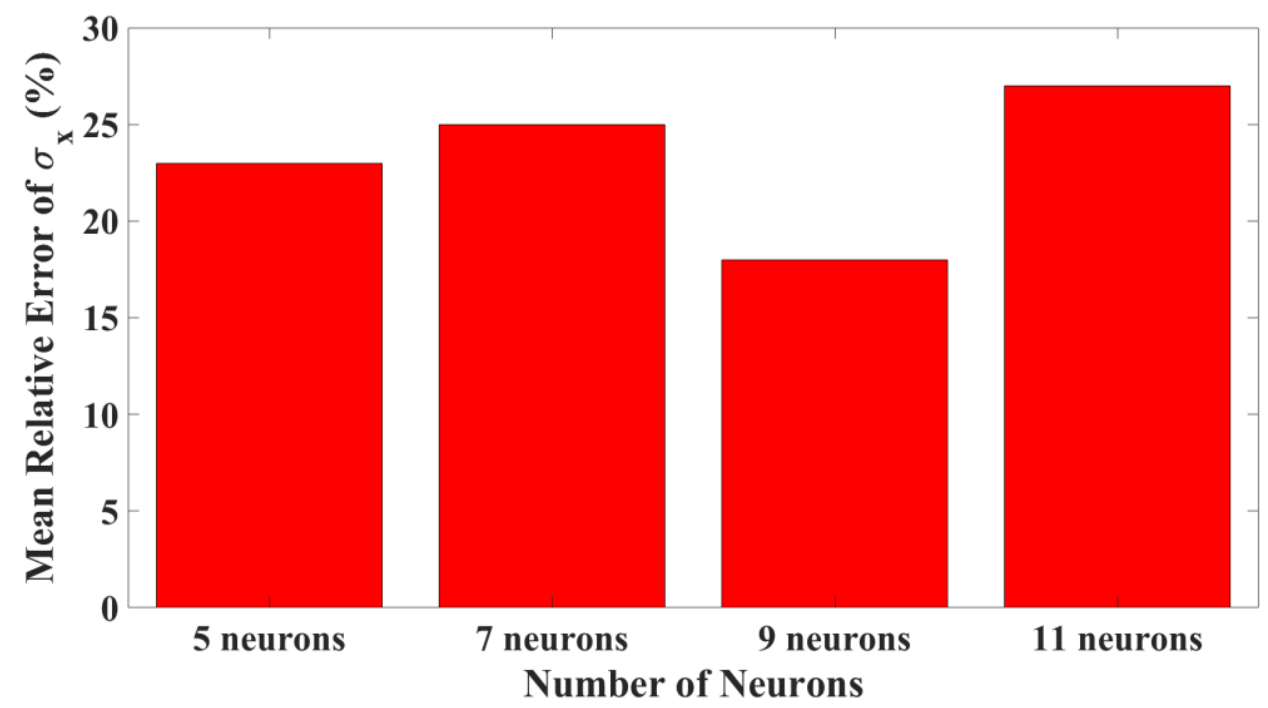

Figure 25. Mean Relative Error for 5, 7, 9 and 11 number of neurons

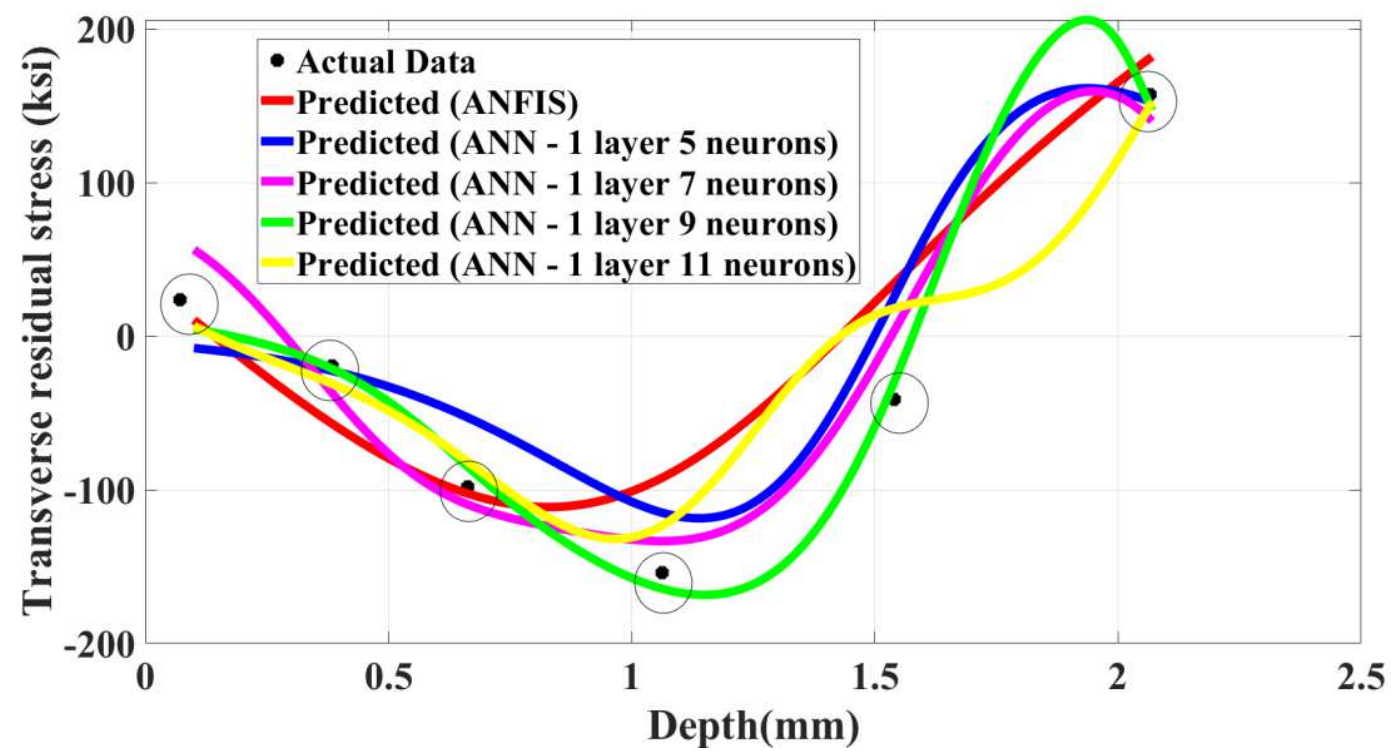

Figure 26. comparison of the ANNs model with different number of neurons and ANFIS with actual data

Figure 25 shows that the least error occurs with a model with 9 neurons, which leads to a 17percent error rate. Figure 25 compares the different number of neurons along with the ANFIS results to the actual numerical data in the middle section of the bead. The actual data is shown by discrete solid black circles. It can be seen that the green-coloured case of 9 neurons aligns 
well with the actual data. Therefore, for the rest of the results one hidden layer with 9 neurons is used for neural network models.

For the sake of better representing the outcome of these ANNs and ANFIS models in the threedimensional domain, the predicted residual stresses and hardness are shown in the middle section of the bead. The following two graphs compare the ANN and ANFIS predictions of the residual stress and hardness to the actual numerical results (Figures 27 and 28). It is clearly illustrated that right at the actual points (emphasized by black circles), the ANN results are closer.

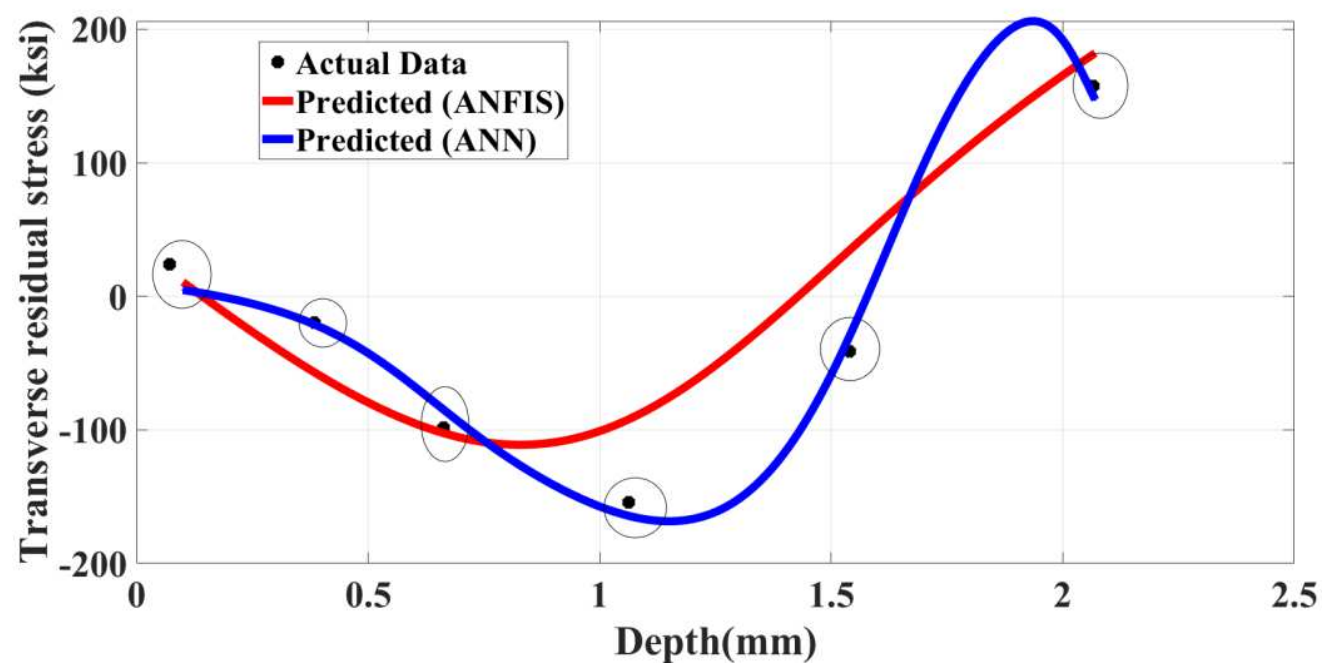

Figure 27. Comparison of ANNs and ANFIS with actual data in the middle section along the depth (transverse residual stress) 


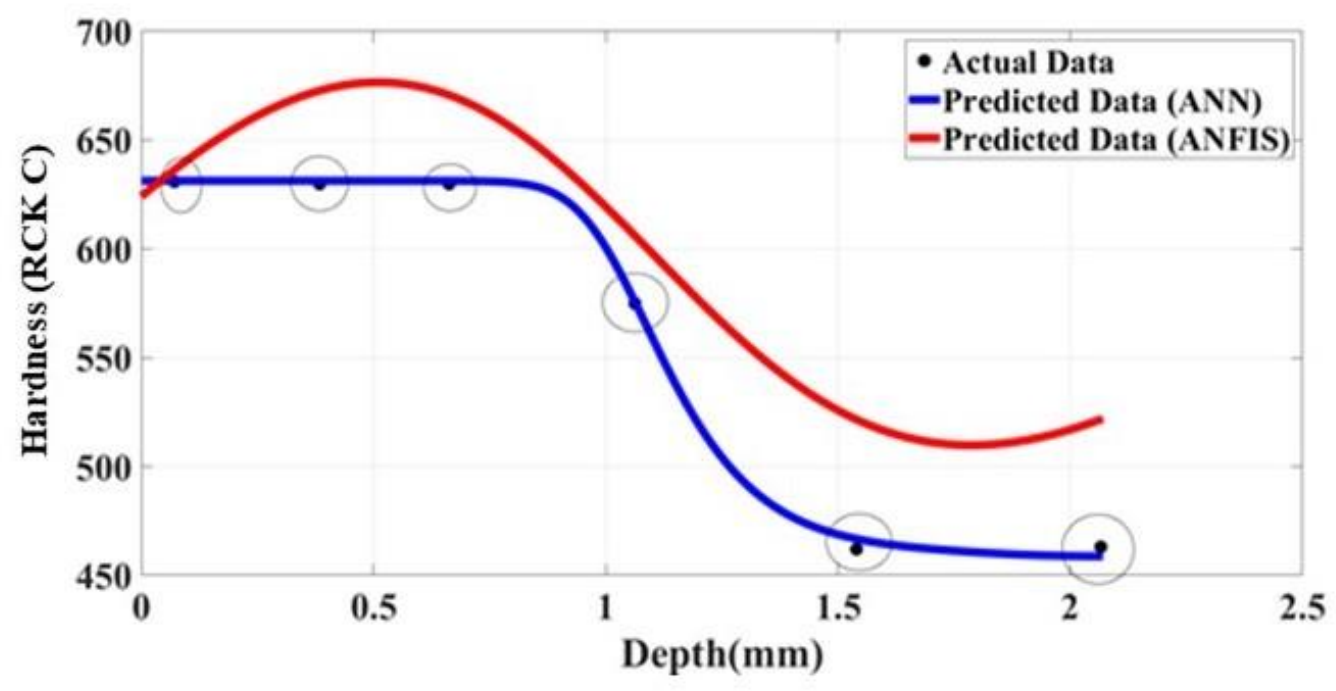

Figure 28. Comparison of ANNs and ANFIS with actual data in the middle section along the depth (hardness)

For the sensitivity analysis, the derivative of transverse residual stress with respect to the length of the bead (z-direction), laser power, and laser speed is considered (Figures 29, 30 and 31). Figure 29 shows that on one hand, the derivative of the residual stress with z-direction in both ANN and ANFIS is zero or near zero. This means that the transverse residual stress does not vary along the length of the bead. In this way, the two-dimensional assumption can be applied to analyse one-bead cases. However, in the case of multi-bead and multi-application of the heat source, the scenario might be different. On the other hand, although the ANFIS shows nearzero values, there is a small variation of the residual stress sensitivity along the length of the bead. 


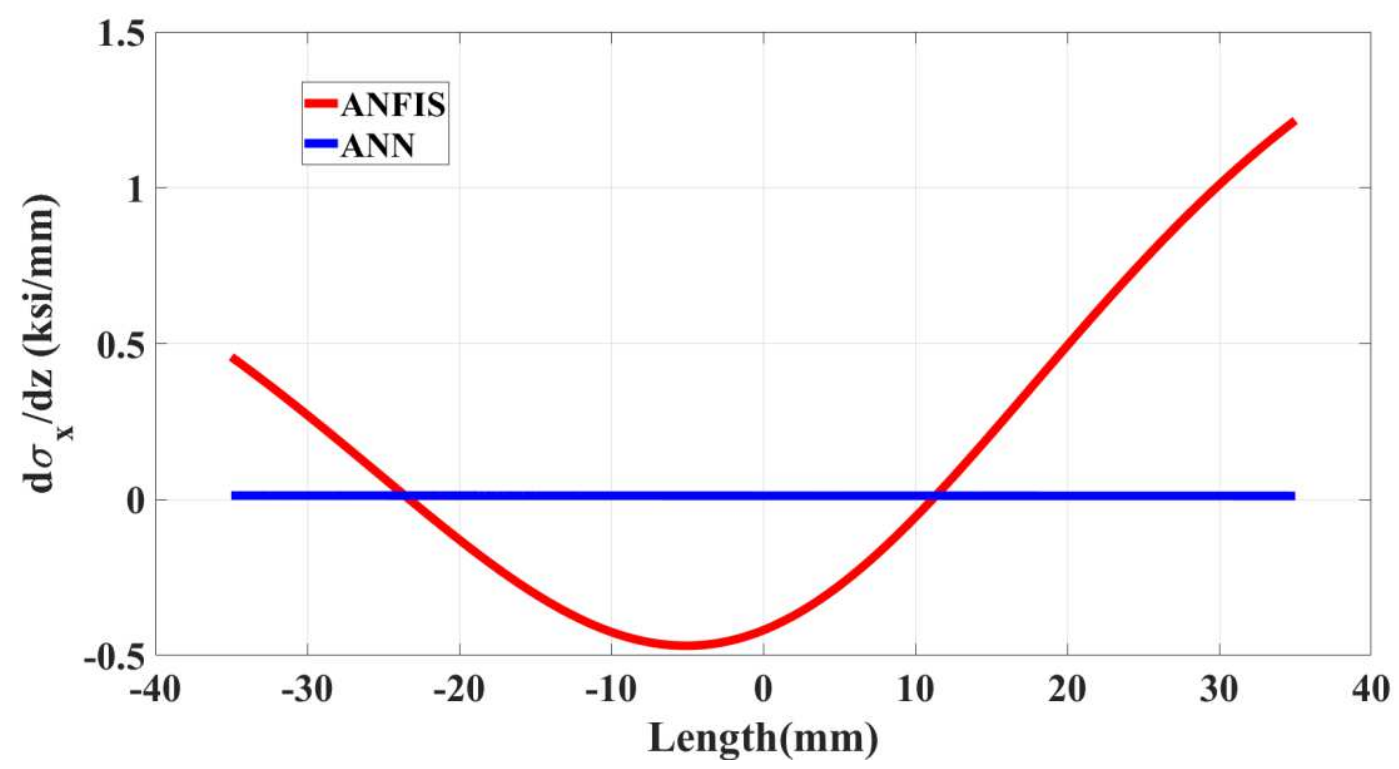

Figure 29. Sensitivity of transvers residual stress with respect to the z-direction

Based on the ANN sensitivity analysis in Figure 30, as the heat energy changes a little, the residual stress can vary up to $110 \mathrm{Ksi}$, and this sensitivity decreases as the applied heat energy rises. It appears that ANFIS approach does not show predictable trends for sensitivity. More research needs to be done to determine whether patterns could emerge.

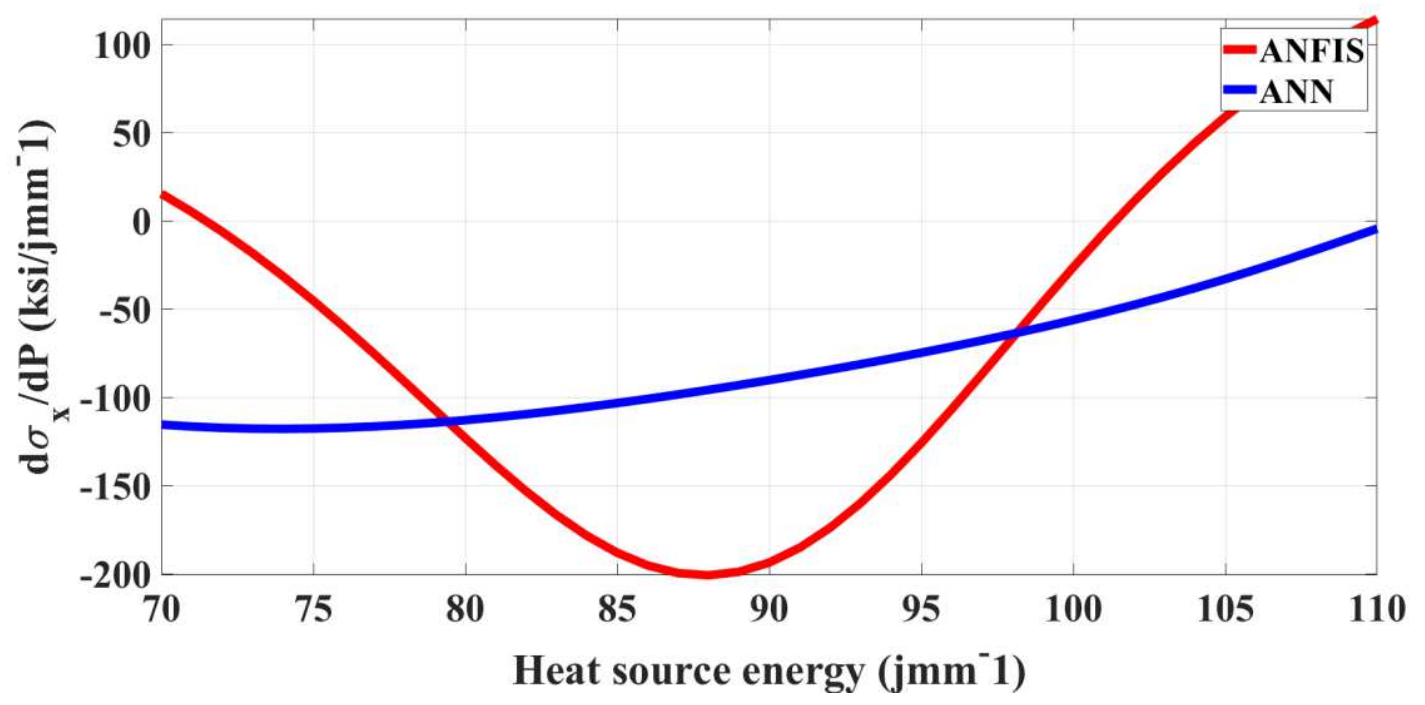

Figure 30. Sensitivity of transvers residual stress with respect to the applied heat energy Figure 31 demonstrates the sensitivity of residual stress with respect to the laser speed. As expected in terms of a physical point of view, transverse residual stresses are affected by the 
laser speed. The ANN results in this figure point that this sensitivity remains constant in different speed levels, while the ANFIS results show variations.

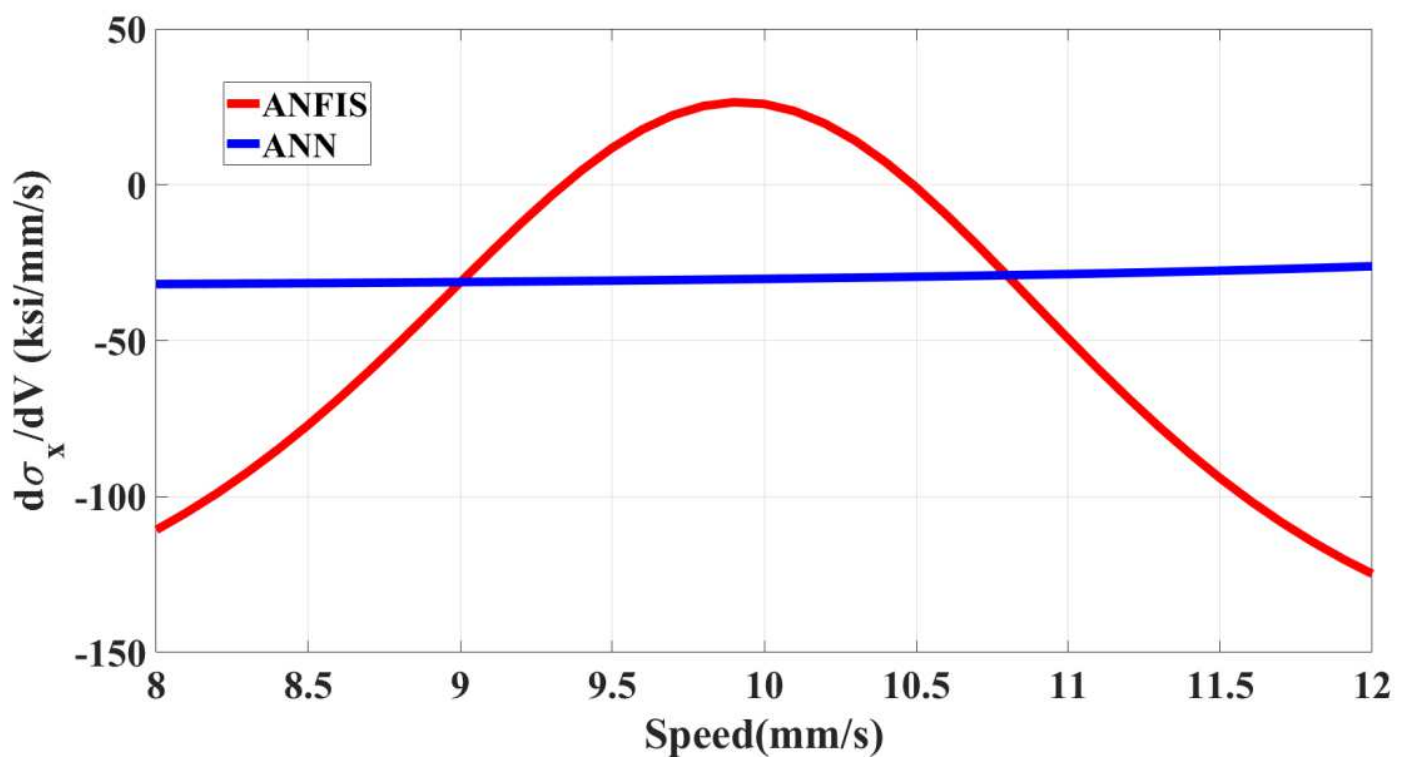

Figure 31. Sensitivity of transvers residual stress with respect to the laser speed

The ANN and ANFIS methods can be used to predict characteristics throughout the bead, but the goodness of the prediction varies between the ANN and ANFIS approaches. The ANFIS model results show similar patterns to the collected data, but do not align well to the collected data points. However, the ANN modelling approach does not appear to have this issue, but there are overshoot regions that are observed. Data sufficiency may be the issue for both approaches. There will be other issues that will arise when developing predictive models for complex multi-bead scenarios, but this research shows the potential with an AI predictive modelling strategy using experimental and simulation data.

\section{Summary and Conclusions}

Much experimental data must be collected to develop a comprehensive prediction models for the DED process, and simulation approaches are computationally intensive. Therefore, machine learning approaches using data from the experimental and simulation domains has 
much potential. Using a data fusion approach, machine learning based predictive models for a single laser clad in the 1D, 2D and 3D domains are explored. Each solution has its unique model structure; therefore, the nature of the problem being considered influences the structure of the solution.

For the 1D domain, discrete geometry and properties are predicted. Averaged values are used for the hardness values as previous analyses have shown that the hardness is consistent in the bead, but changes in the dilution and heat affected zones. The residual stress varies throughout the bead. This shows the need for a 2D or 3D approach for this property as well. Interestingly, with minimal data the model predictions are generally accurate for all the parameters being assessed; however, the prediction data is limited in scope. More data will improve the model accuracy, but the limitations will remain.

When assessing the residual stress model in the 2D domain (the bead cross-sections), the ANFIS model generated less error. The prediction has good accuracy but there might be the chance of missing the maximum residual stress since the analysis is only for one cross-section of the bead. It cannot be assumed that the residual stress patterns in the center of the bead are consistent throughout. Therefore, the study is expanded to the 3D domain, where the residual stress values along with the bead predicted. This data included variability throughout the data set. Although, the ANN and ANFIS models are capable of prediction with very good accuracy issues related to both solution approaches are raised. Data sufficiency is one issue as properties vary between the nodes, as shown in Fig. 8 and 9. Training a neural network with nondimensional geometry parameters could lead to more comprehensive results, and this is future work. This research can be expanded to multiple bead scenarios with different percentage overlaps and bead stacking, which introduces another level of complexity. 


\section{Acknowledgements}

The support provided by MITACs, CAMufacturing Solutions, Inc., and Lincoln Laser Inc. are gratefully acknowledged.

\section{Authors' contributions}

BM: Simulation model setup, data collection, analysis, developing mathematical model and paper write up. SEM: Developing mathematical model and paper write up. AP: Developing mathematical model and paper write up. RJU: Experimental data collection and analysis, simulation data analysis, research paper writing and editing, project funding

\section{Funding}

This research was funded by Mitacs Accelerate. Award number: IT18391, IT16398 . Grant recipients: Dr. Jill Urbanic, Dr. Ofelia Jianu and also the NSERC grant, award number: RGPIN/04842-2018, Grant recipients: Dr. Jill Urbanic.

Data and material availability Not applicable

Code availability Not applicable

\section{Declarations}

\section{Ethics approval}

The manuscript is not submitted to any other journal simultaneously. The manuscript will not be submitted elsewhere until the editorial process is completed. The submitted work is original. A single study "Machine Learning Approaches for Predicting Geometric and Mechanical Characteristics for Single P420 Laser Beads Clad onto an AISI 1018 Substrate" has not been split up into several parts to increase the quantity of submissions. There is not any content being translated from other journals in other languages. Results are presented clearly, honestly, and without fabrication, falsification or inappropriate data manipulation. No data, text, or theories by others are presented as if they were the author's own ("plagiarism"). The authors have 
permission to use the applied software. This research has not been applied to pose a threat to public health or national security.

\section{Consent to participate}

Not applicable

\section{Consent for publication}

All authors consent to publish the paper in the current order. The authors transfer to Springer the non-exclusive publication rights.

\section{Conflict of interest}

The authors declare that they have no conflict of interest.

\section{References}

[1] M. Srinivas and B. S. Babu, "A Critical Review on Recent Research Methodologies in Additive Manufacturing," in Materials Today: Proceedings, Jan. 2017, vol. 4, no. 8, pp. 9049-9059, doi: 10.1016/j.matpr.2017.07.258.

[2] A. Emamian, S. F. Corbin, and A. Khajepour, "Effect of laser cladding process parameters on clad quality and in-situ formed microstructure of Fe-TiC composite coatings," Surface and Coatings Technology, vol. 205, no. 7, pp. 2007-2015, Dec. 2010, doi: 10.1016/j.surfcoat.2010.08.087.

[3] J. Shi, P. Zhu, G. Fu, and S. Shi, "Geometry characteristics modeling and process optimization in coaxial laser inside wire cladding," Optics and Laser Technology, vol. 101, pp. 341-348, May 2018, doi: 10.1016/j.optlastec.2017.10.035.

[4] M. K. Alam, R. J. Urbanic, N. Nazemi, and A. Edrisy, "Predictive modeling and the effect of process parameters on the hardness and bead characteristics for laser-cladded stainless steel," The International Journal of Advanced Manufacturing Technology, vol. 94, no. 1, pp. 397-413, 2018, doi: 10.1007/s00170-017-0898-5.

[5] K. Aggarwal, R. J. Urbanic, and S. M. Saqib, "Development of predictive models for effective process parameter selection for single and overlapping laser clad bead geometry," Rapid Prototyping Journal, 2018.

[6] T. Chen, W. Wu, W. Li, and D. Liu, "Laser cladding of nanoparticle TiC ceramic powder: Effects of process parameters on the quality characteristics of the coatings and 
its prediction model," Optics \& Laser Technology, vol. 116, pp. 345-355, 2019, doi: https://doi.org/10.1016/j.optlastec.2019.03.048.

[7] P. Zareh and R. J. Urbanic, "Experimental analysis of single layer multi-track deposition of clad beads with variable overlap percentages," The International Journal of Advanced Manufacturing Technology, vol. 109, no. 5, pp. 1511-1525, 2020, doi: 10.1007/s00170-020-05672-5.

[8] Y. Zhao, C. Guan, L. Chen, J. Sun, and T. Yu, "Effect of process parameters on the cladding track geometry fabricated by laser cladding," Optik, vol. 223, p. 165447, 2020, doi: https://doi.org/10.1016/j.ijleo.2020.165447.

[9] E. Mirkoohi, D. E. Sievers, H. Garmestani, and S. Y. Liang, "Thermo-mechanical modeling of thermal stress in metal additive manufacturing considering elastoplastic hardening," CIRP Journal of Manufacturing Science and Technology, vol. 28, pp. 5267, Jan. 2020, doi: 10.1016/j.cirpj.2020.01.002.

[10] N. Nazemi, J. Urbanic, and M. Alam, "Hardness and residual stress modeling of powder injection laser cladding of P420 coating on AISI 1018 substrate," The International Journal of Advanced Manufacturing Technology, vol. 93, no. 9, pp. 3485-3503, 2017, doi: 10.1007/s00170-017-0760-9.

[11] E. R. Denlinger, M. Gouge, J. Irwin, and P. Michaleris, “Thermomechanical model development and in situ experimental validation of the Laser Powder-Bed Fusion process," Additive Manufacturing, vol. 16, pp. 73-80, Aug. 2017, doi: 10.1016/j.addma.2017.05.001.

[12] R. J. Williams, C. M. Davies, and P. A. Hooper, “A pragmatic part scale model for residual stress and distortion prediction in powder bed fusion," Additive Manufacturing, vol. 22, pp. 416-425, Aug. 2018, doi: 10.1016/j.addma.2018.05.038.

[13] B. Kemerling, J. C. Lippold, C. M. Fancher, and J. Bunn, "Residual stress evaluation of components produced via direct metal laser sintering," doi: 10.1007/s40194-0180572-z.

[14] S. Chowdhury and S. Anand, "Artificial neural network based geometric compensation for thermal deformation in additive manufacturing processes," 2016.

[15] F. Caiazzo and A. Caggiano, "Laser direct metal deposition of 2024 Al alloy: trace geometry prediction via machine learning," Materials, vol. 11, no. 3, p. 444, 2018.

[16] K. Ren, Y. Chew, Y. F. Zhang, J. Y. H. Fuh, and G. J. Bi, "Thermal field prediction for laser scanning paths in laser aided additive manufacturing by physics-based 
machine learning," Computer Methods in Applied Mechanics and Engineering, vol. 362, p. 112734, 2020.

[17] A. K. Sood, R. K. Ohdar, and S. S. Mahapatra, "Experimental investigation and empirical modelling of FDM process for compressive strength improvement," Journal of Advanced Research, vol. 3, no. 1, pp. 81-90, Jan. 2012, doi: 10.1016/j.jare.2011.05.001.

[18] R. P. Singh, "Analysis of Depth of Penetration and Impact Strength during Shielded Metal Arc Welding under Magnetic Field using Artificial Neural Networks."

[19] Q. Wu, T. Mukherjee, A. De, and T. DebRoy, "Residual stresses in wire-arc additive manufacturing - Hierarchy of influential variables," Additive Manufacturing, vol. 35, p. 101355, Oct. 2020, doi: 10.1016/j.addma.2020.101355.

[20] J. Mathew, J. Griffin, M. Alamaniotis, S. Kanarachos, and M. E. Fitzpatrick, "Prediction of welding residual stresses using machine learning: Comparison between neural networks and neuro-fuzzy systems," Applied Soft Computing Journal, vol. 70, pp. 131-146, Sep. 2018, doi: 10.1016/j.asoc.2018.05.017.

[21] M. K. Alam, A. Edrisy, and J. Urbanic, "Microstructural analysis of the laser-cladded AISI 420 martensitic stainless steel," Metallurgical and Materials Transactions A, vol. 50, no. 5, pp. 2495-2506, 2019.

[22] M. A. Denai, F. Palis, and A. Zeghbib, “ANFIS based modelling and control of nonlinear systems: a tutorial," in 2004 IEEE International Conference on Systems, Man and Cybernetics (IEEE Cat. No. 04CH37583), 2004, vol. 4, pp. 3433-3438.

[23] E. Rezaei, A. Karami, T. Yousefi, and S. Mahmoudinezhad, "Modeling the free convection heat transfer in a partitioned cavity using ANFIS," International Communications in Heat and Mass Transfer, vol. 39, no. 3, pp. 470-475, 2012, doi: 10.1016/j.icheatmasstransfer.2011.12.006.

[24] M. Hayati, A. M. Rashidi, and A. Rezaei, "Prediction of grain size of nanocrystalline nickel coatings using adaptive neuro-fuzzy inference system," Solid State Sciences, vol. 13, no. 1, pp. 163-167, 2011, doi: 10.1016/j.solidstatesciences.2010.11.007.

[25] W. Pedrycz, "Why triangular membership functions?," Fuzzy sets and Systems, vol. 64, no. 1, pp. 21-30, 1994.

[26] B. Mohajernia, R. J. Urbanic, and N. Nazemi, "Predictive Modelling of Residual Stresses for Single Bead P420 Laser Cladding onto an AISI 1018 Substrate," IFACPapersOnLine, vol. 52, no. 10, pp. 236-241, 2019. 